\title{
Radiative Properties of Irregular Open Cell Solid Foams
}

\author{
Salvatore Cunsolo $^{1 *}$, Rémi Coquard ${ }^{2}$, Dominique Baillis ${ }^{1}$, Wilson K.S. Chiu ${ }^{3}$, Nicola Bianco ${ }^{4}$ \\ 1. LaMCoS, INSA-Lyon, UMR CNRS 5259, 18-20 Rue des Sciences, F-69621 Villeurbanne, France \\ 2. EC2-MODELISATION, 66 Boulevard Niels Bohr, F-69603 Villeurbanne, France \\ 3. Department of Mechanical Engineering, University of Connecticut, Storrs, CT, USA \\ 4. Dipartimento di Ingegneria industriale, Università degli Studi Federico II, P.le Tecchio 80, 80125, \\ Napoli, Italy \\ *Corresponding author, email: salvatore.cunsolo@insa-lyon.fr
}

\begin{abstract}
$\underline{\text { Abstract }}$
In recent years, Monte Carlo numerical methods have known increasing utilization to characterize radiative properties of open cell solid foams. A great interest has been also observed in recent literature in methodologies to digitally generate foam-like structures to be used in Monte Carlo simulations. These approaches enable researchers to overcome the limitations of studies based on tomographic data. In this work, a novel methodology for generation of foam-like structures is presented. The method is based upon Voronoi partitions with random seeding points, combined with Surface Evolver to obtain a more realistic cell structure. The detailed geometrical characteristics of the struts are taken into account. The generated structures can be created to compare well to real tomographic samples. A number of characteristics of the resulting structure can be controlled. The application of Monte Carlo simulations to the generated structures allows the precise evaluation of each parameter' influence on the extinction coefficient. This in turn makes it possible to propose some simplified analytical
\end{abstract}


correlations. The correlations are validated against Monte Carlo simulations on tomographic data and compared with existing reference relations from literature. Finally, simplified forms of the relations are proposed.

Keywords: Open cell foams, Radiation, Monte Carlo, Digital generation, Voronoi, Analytical laws

\section{NOMENCLATURE}

\section{Latin symbols}

CV Coefficient of variation of cell diameter

$d \quad$ Local strut diameter, $m$

$d_{c} \quad$ Cell diameter (equivalent sphere), $m$

$d_{c, G} \quad$ Cell diameter (equivalent dodecahedron), $m$

$d_{i} \quad$ Strut cross section incircle diameter

$d_{j, \max } \quad$ Strut junction maximum diameter, $m$

$d_{\max } \quad$ Maximum strut diameter, $m$

$d_{\min } \quad$ Minimum strut diameter, $m$

$d_{o} \quad$ Strut cross section circumcircle diameter, $m$

$g \quad$ Phase function asymmetry factor

$I / I_{0} \quad$ Unextincted fraction of radiation

$k \quad$ Normalized curvature

$l \quad$ Strut length, local abscissa, $m$

$L \quad$ Total strut length, $m$

$N \quad$ Number of rays

$p_{\text {avg }} \quad$ Mean free path, $m$

$p_{n} \quad$ Free path of the $\mathrm{n}$-th ray, $\mathrm{m}$

$S_{s} \quad$ Strut cross section surface, $\mathrm{m}^{2}$

$S_{v} \quad$ Specific surface area, $m^{-1}$

$t \quad$ Diameter ratio

\section{Greek symbols}

$\beta \quad$ Extinction coefficient $\left(\mathrm{m}^{-1}\right)$

$\beta^{+} \quad$ Nondimensional extinction coefficient

$\varepsilon \quad$ Porosity

$\Phi(\mu) \quad$ Scattering phase function

$\rho_{s} \quad$ Solid surface reflectivity

$\sigma_{d_{c}} \quad$ Standard deviation of cell diameter, $m$

$\omega \quad$ Scattering albedo 


\section{Introduction}

Cellular foams are a key material for many technological applications. Their high porosity (or low relative density) and large specific surface area play an important role from the thermal point of view. For example, high porosity closed cell polymer foams are used as efficient insulating materials [1-4]. Metal or ceramic foams are being employed in a variety of high temperature applications, such as volumetric solar energy receivers for CSP plants [5], compact heat exchangers [6], porous radiant burners [7, 8] and fire barriers [9]. Accurate modeling of thermal properties is obviously highly desirable for the optimization of the performance in these applications. Considering the high porosity (typically in a range from $85 \%$ up to $98 \%$ ), radiative heat transfer contribution can be significant, and in some cases even prevalent over other heat transfer modes [10]. For this reason, a large number of analytical and numerical approaches have been dedicated to the characterization of radiative heat transfer in cellular solid foams. Most studies focus on determining appropriate equivalent continuous medium properties.

Specifically focusing on radiation, the current baseline state-of-the-art approach is based on the utilization of the Radiative Transfer Equation (RTE). General lines on the usage of RTE for radiative transfer can be found for example in textbooks [11-14]. While alternative approaches exist, the RTE approach is usually considered sufficiently accurate for most practical cases, if the relevant coefficients (radiative properties) are correctly determined [15-17].

As such, most of the literature has been focused on finding efficient and reliable ways to determine radiative properties [18-20]. In recent years, numerical methods based on Monte Carlo techniques for the determination of radiative properties are becoming established in order to study either real structures obtained from tomographic imaging or computer generated structures that closely mimic the microstructure of the real foams. 
Tancrez and Taine [21] proposed to use the Radiative Distribution Function Identification (RDFI) model and determined radiative properties of spherical packed beds. Zeghondy et al. [22, 23] and Petrasch et al. [24] applied the RDFI approach to tomographic data of cellular foam samples. Coquard et al. [25-27] proposed to use an alternative Monte Carlo approach based on mean free path calculation. Cunsolo et al. [28] recently presented a review including an extensive discussion of numerical methods, including Monte Carlo techniques.

Techniques fully based on tomographic data provide satisfactory agreement with experimental data, but their dependence on high quality scans of existing foam samples makes them of limited utility for design purposes. To overcome these limitations, a number of recent studies have sought to digitally reproduce the foam structures using different approaches, including mathematical morphology operations applied on existing tomography data [29, 30], simulation of the bubbling process [31], regular [32, 33] and irregular [34, 35] Voronoi partitions. By computer generating a number of structures and running numerical simulations $[29,30,35]$ it is possible to obtain useful results for the optimization of energy transfer. Irregular 3D Voronoi structures seem to be particularly promising for this purpose as they can be described with a limited amount of parameters, are based on well-known generation methods and approximate the structures of real foam reasonably well [36].

In the current paper, a novel methodology is presented that allows the generation of polygonal mesh to represent high porosity open cell foams with high control of a number of geometrical parameters. The methodology is subsequently applied systematically with individually varying parameters to generate a number of structures. The generated structures are introduced into a Monte Carlo algorithm for the calculation of radiative properties, and especially of the extinction coefficient, $\beta$. For each structure, the specific surface area $S_{v}$ and porosity $\varepsilon$ are also calculated, and the normalized extinction coefficient $\beta^{+}$ $=4 \beta \cdot \varepsilon / S_{v}[21]$ is deduced. This procedure makes it possible to determine which parameters have a 
significant effect on the extinction coefficient and which parameters have a negligible effect, thus defining the inputs required to calculate the extinction coefficient with a given accuracy. Based on this assessment, new analytical relations are given that fit the numerical results with a minimum number of parameters and with more accuracy than those usually used in the literature. These relations are expected to be useful for material design purposes.

\section{$2 \quad$ Methodology}

\subsection{Digital generation methodology}

\subsubsection{Digital generation methodology - Presentation}

In the present work, a methodology is proposed that makes it possible to generate realistic foam structures. The methodology involves the generation of a pseudo-random lattice of seeding points, the generation of a Voronoi diagram of these points, the stabilization of the resulting cell structure in Surface Evolver [37], the addition of polygonal struts along the resulting skeleton, and finally the virtual welding of the polygonal struts at their intersections through a shrink-wrapping [38] process. While similar generation methods based on Voronoi diagrams have already been presented in literature $[28,34,35]$, the current approach does present some distinct features, specifically the use of Surface Evolver and the capability to directly generate intersection-free triangular meshes.

The process requires initially generating a number of seeding points. The corresponding final structure will be a periodic structure containing as many cells as initial seeding points. For this study, a number of 128 initial seeding points, corresponding to 128 cells final structure has been used. This number of cells ensures convergence of Monte Carlo algorithms [28] and allows creating a cubic Kelvin foam (see section 3) with a whole number of Kelvin periodic units (made up by 2 cells). The points are generated with a Random Sequential Absorption algorithm [39] that drops equal-sized spheres into space, 
enforcing non-overlapping condition, with a final packing density around $30 \%$, the centers of the spheres being used as the seeding point. This serves to insure a minimum distance between any two seeding points [Fig. 1].
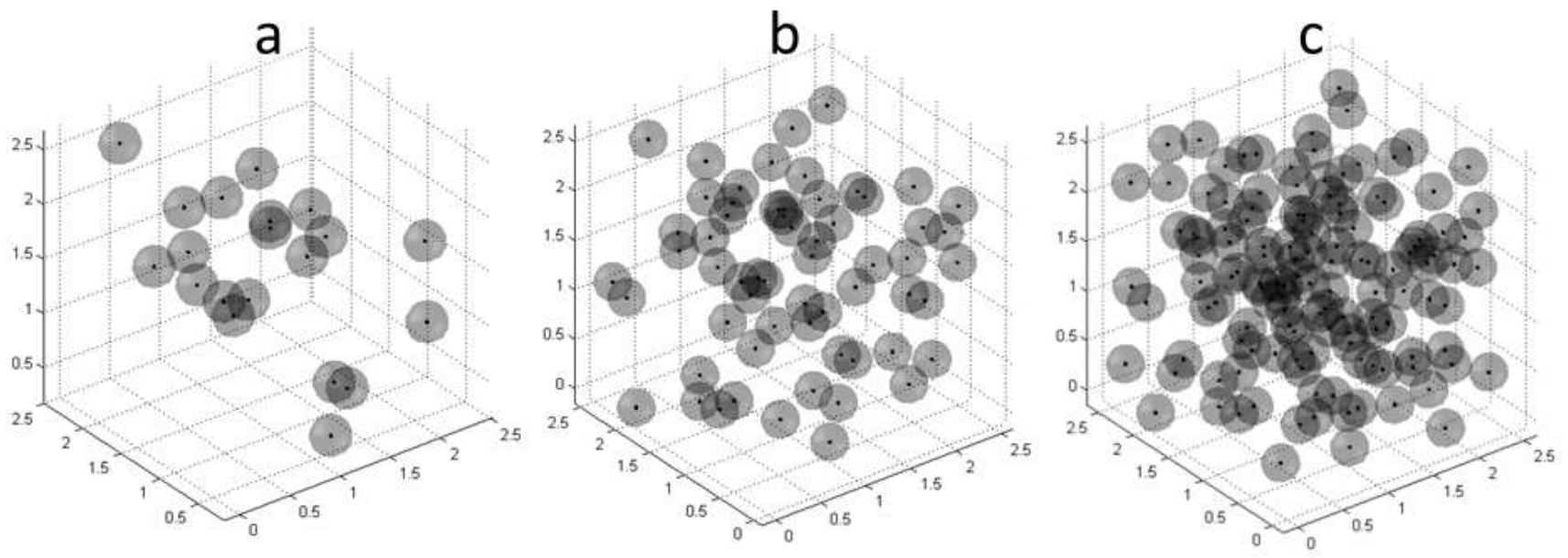

Fig. 1 - Progressive dropping of spheres into space at 5\% (a), 16\% (b) and 30\% (c) packing density.

The periodic 3D Voronoi diagram of the seeding points is then generated. The Voronoi diagram partitions the space in polyhedral regions, one for each seeding point. Every point of a given region is closer to that region's seed than to any other seed. The resulting data structure is adapted into a Surface Evolver input file. Treating the structure with Surface Evolver makes it possible to achieve two results:

- The resulting structure is an energetically stable structure rather than just a random structure, that which should make it more realistic.

- The cell size distribution can be directly specified and enforced in Surface Evolver, rather than indirectly as in approaches using perturbed regular structures [28, 35]

The structure is refined and processed in Surface Evolver until a stabilized final structure is obtained. The size distribution of cells used are Gaussian unimodal distributions of varying standard deviation. The dispersion of the cell size distribution is controlled through its coefficient of variation $C V=\sigma_{d_{c}} /$ 
$\overline{d_{c}}$. Edges with three neighboring cells, i.e. Plateau edges, are then isolated to obtain the structure's skeleton, used in further processing [Fig. 2].
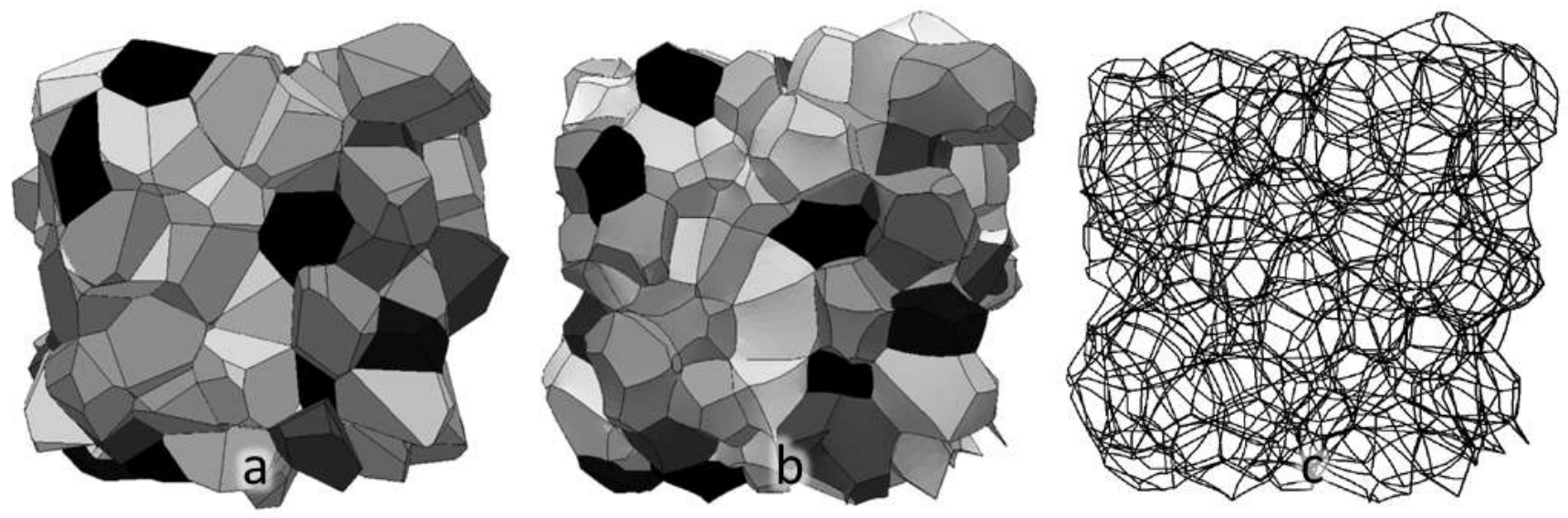

Fig. 2 - (a) Initial cell structure (Voronoi diagram) (b) Processed cell structure (after Surface Evolver)

(c) Cell structure skeleton.

In further processing, polygonal struts are added along the edges. The strut cross section is represented as an equilateral triangle with curved sides of constant curvature. The shape of the curved sides can be varied, giving rise to concave, flat or round shapes. The shape of the triangle is described using a single parameter $k$, labeled normalized curvature, which corresponds to the curvature radius of the circle circumscribing of the triangle divided by the local signed curvature radius of the sides. For values of $-1 / \sqrt{3} \leq k \leq 1$ the cross-section varies from maximally concave triangular, to flat triangular, to circular [Fig. 3]. 


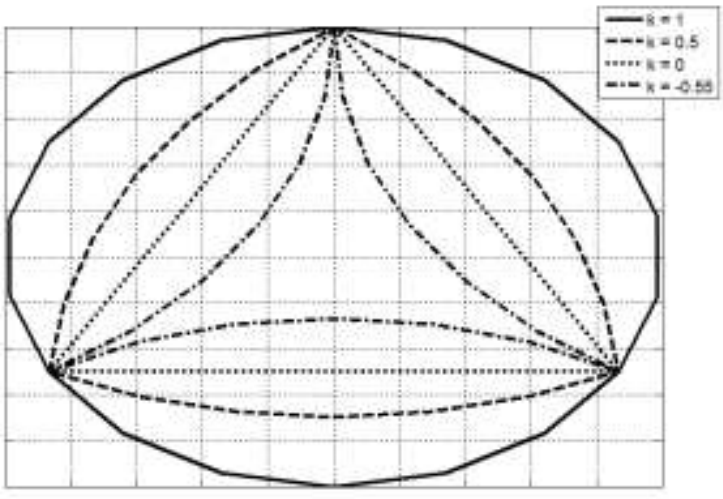

Fig. 3 - Cross section of the strut

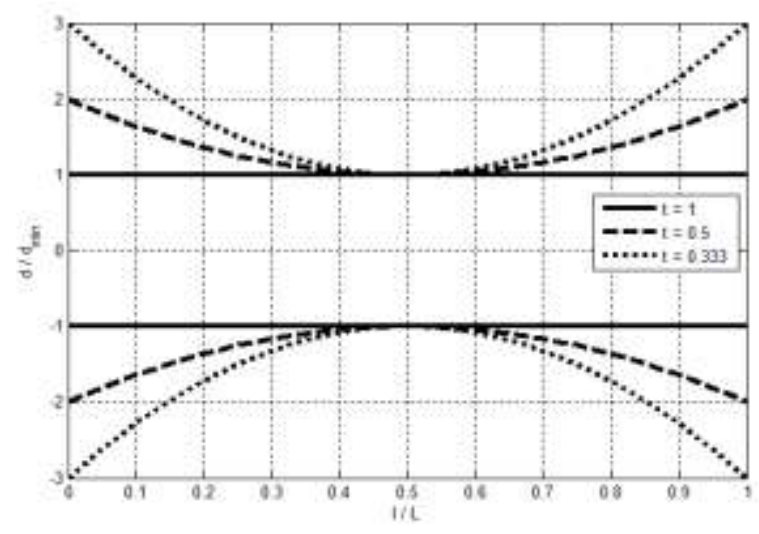

Fig. 4 - Longitudinal profile of the strut

Additionally, the longitudinal profile of the struts varies according to a quadratic law, i.e. the diameter of the circle circumscribing the cross section varies according to a law $d=d_{\min }\left[1+\frac{(1-t)(2 l-L)^{2}}{t L^{2}}\right]$ where $0 \leq l \leq L$ is the local abscissa and $L$ is the length of the strut. The ratio of minimum to maximum diameter, $t=d_{\min } / d_{\max }$ can be controlled [Fig. 4].

The resulting structure constituted by all the struts together is not a continuous mesh, but rather presents a number of self-intersections, namely at the junction points of the struts. The application of a "virtual welding" (shrink-wrapping) process at the junction makes it possible to obtain a continuous mesh, free of self-intersections. This process involves identification of intersecting facets, construction of the convex hull of said facets, then iterative refining and projection of said convex hull into the original polygonal structure, and smoothing of the resulting mesh [38]. Fig. 5 depicts a typical junction before and after the process.

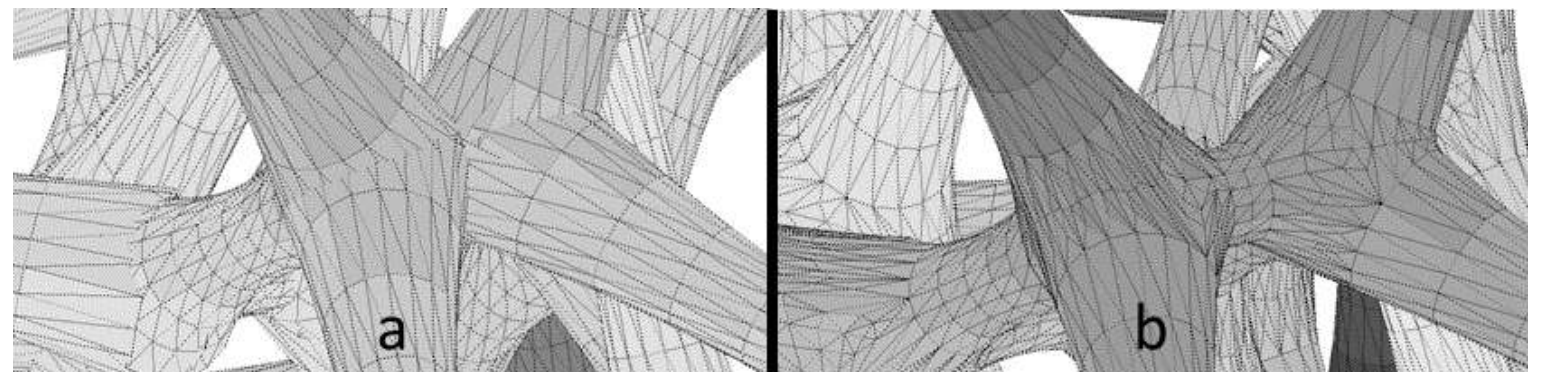

Fig. 5 - Structure before (a) and after (b) the "virtual welding" process. 
As a final step, the structures are cut along their periodic bounding box to obtain cubic, periodic representation [Fig. 6].

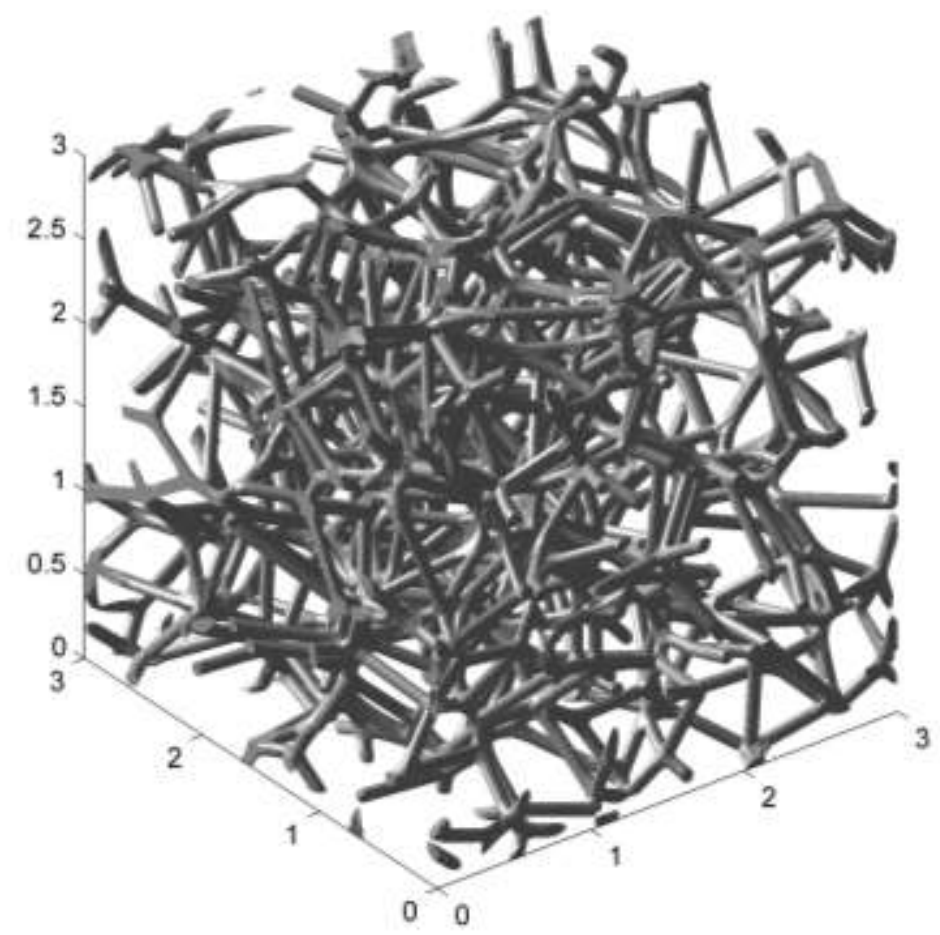

Fig. 6 - Example of finalized structure $(C V=5 \% ; t=1 ; \varepsilon=94 \% ; k=1)$

The present algorithm presents two distinct features:

1) It makes use of the software Surface Evolver to obtain a more realistic, stabilized cell structure with a finely controlled final cell size distribution.

2) It directly generates the triangular mesh without ever resorting to a voxel representation of the structure, that which allows representing very fine details in large structures.

The second feature of the algorithm is of paramount importance for the feasibility of the next part of the study, where to provide a more realistic description of the structure, both sub-pore scale geometrical features (e.g. strut cross section) and pore scale geometrical features (e.g. pore size distribution dispersion) are considered. 


\subsubsection{Digital generation methodology - Validation}

To verify the capabilities of the proposed methodologies, digitally generated structures have been compared with data obtained through tomography of real metal foam samples. Tomographic data was acquired for four samples by means of micro computed tomography $(\mu \mathrm{CT})$. General information about the samples is presented in Tab. 1. Samples 1 and 2 have been used in previous work of the authors, while samples 3 and 4 are new experimental material, introduced to have a better coverage of the typical range of porosities of metal foams.

\begin{tabular}{|c|c|c|c|c|}
\hline Sample number & Material & Nominal porosity & Nominal PPI & $\mu$ CT resolution \\
\hline $1[25]$ & $\mathrm{Al}-\mathrm{NiP}$ & $90 \%$ & 60 & $30 \mu \mathrm{m}$ \\
\hline $2[32]$ & $\mathrm{Al}$ & $90 \%$ & 40 & $44 \mu \mathrm{m}$ \\
\hline 3 & $\mathrm{Al}$ & $94 \%$ & 40 & $22 \mu \mathrm{m}$ \\
\hline 4 & $\mathrm{Al}$ & $97 \%$ & 40 & $22 \mu \mathrm{m}$ \\
\hline
\end{tabular}

Tab. 1 - Characteristics of the samples considered.

The tomographic data has been analyzed using the free software iMorph [40] to extract the equivalent cell diameter distribution and the cell connectivity distribution. The cell connectivity represents the number of neighbor cells for each cell and can be thought of as akin to the number of faces per cell.

The equivalent cell diameter distributions show a Gaussian unimodal shape with mean values of $d_{c}=$ $\{1842 ; 2431 ; 2892 ; 2725\} \mu \mathrm{m}$ and coefficients of variations $C V=\sigma_{d_{c}} / \overline{d_{c}}=\{3.46 \% ; 2.46 ; 3.39 ; 4.34 \%\}$ respectively for the four samples [Fig. 7]. The connectivity distributions show a similarly Gaussian shape, with some skew; however, the average value (around 12) appears to be very similar among the samples considered [Fig. 8].

Corresponding digital structures have been generated for each real sample, trying to match mean value and coefficient of variation of the equivalent cell diameter distribution. It is worth noting that the matching has not been obtained by means of an iterative process, but rather by directly inputting the desired quantities in the algorithm. Results appear to be satisfying and are shown in Fig. 9. 
Quantitatively, the original distributions and their matched distributions show a shared area fraction [Fig. 9] of $f=A_{\text {shared }} / A_{\text {tot }}=\{92 \% ; 87 \% ; 96 \% ; 95 \%\}$ respectively for the four samples. Chi-squared goodness-of-fit tests between real and matched distributions result in values of $p=\{0.92$; $0.77 ; 0.998 ; 0.98\}$ respectively for the four samples.

Additionally, the connectivity distributions of generated and real structures have been compared: considering that this distribution does not appear to vary very much between samples, an averaged distribution has been considered. The results appear satisfying [Fig. 10]. Quantitatively, the two distributions present a shared area fraction of $f=91 \%$. Chi-squared test goodness-of-fit tests between the real and the matched distribution result in $p=0.25$.

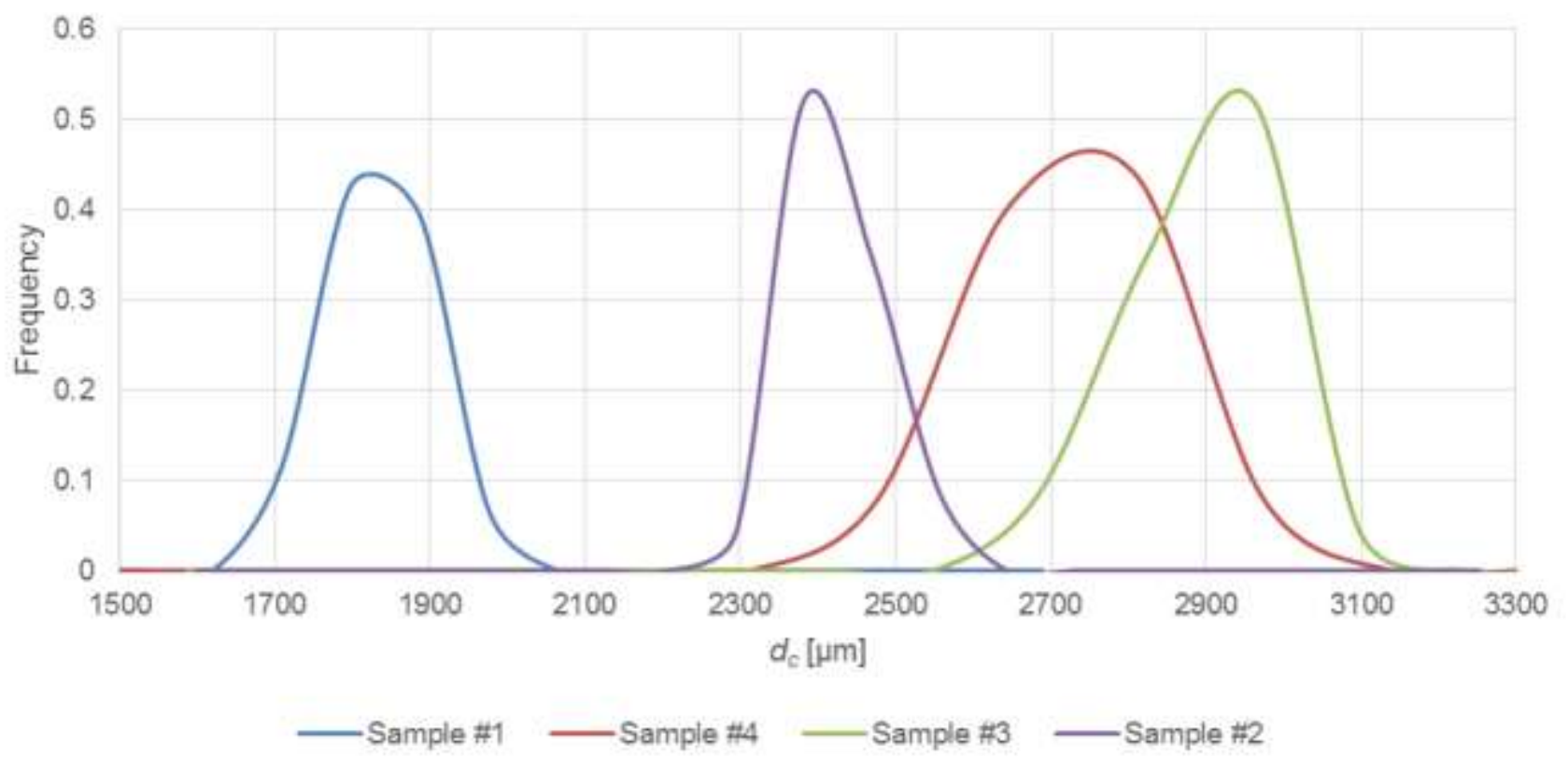

Fig. 7 - Equivalent cell diameter distributions of the 4 samples, as calculated by iMorph. 


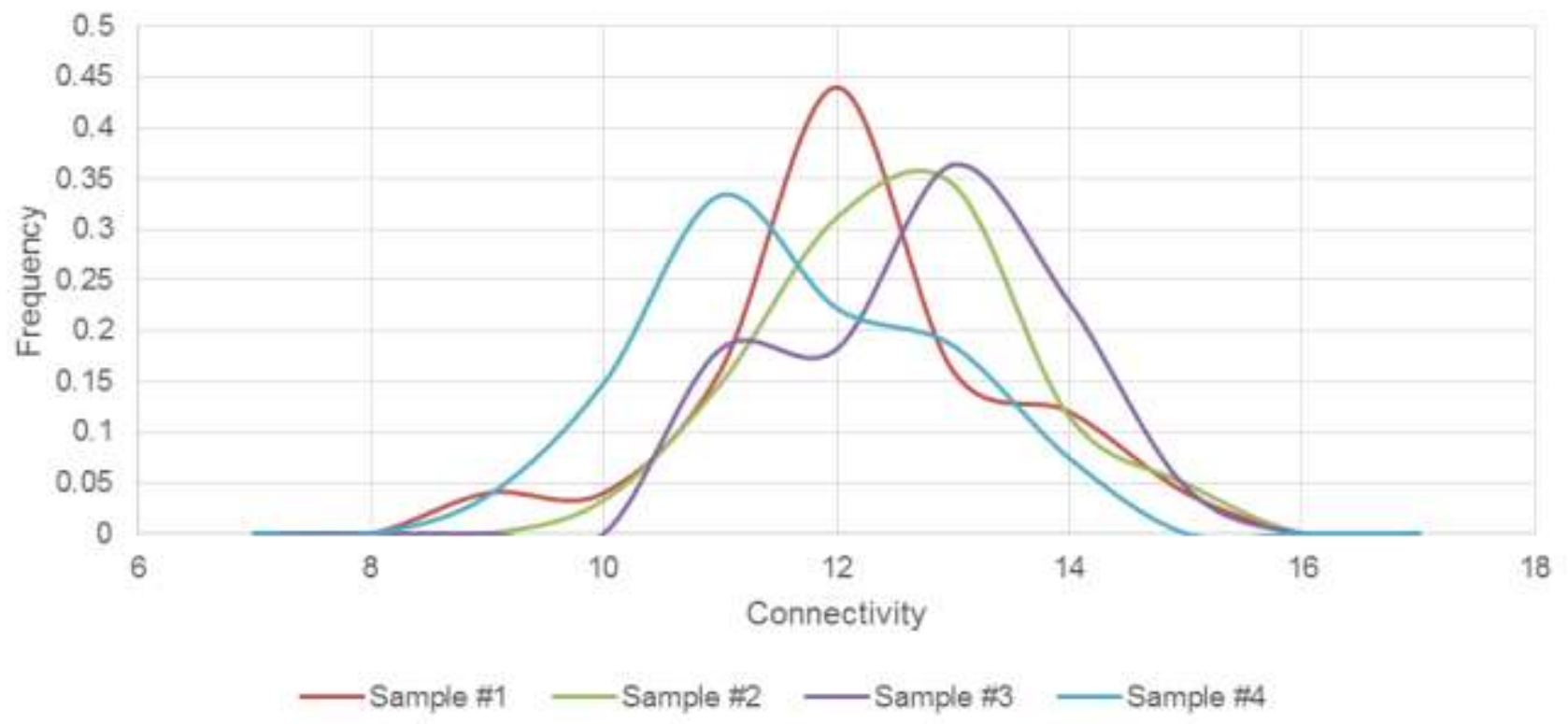

Fig. 8 - Cell connectivity distribution of the 4 samples, as calculated by iMorph.

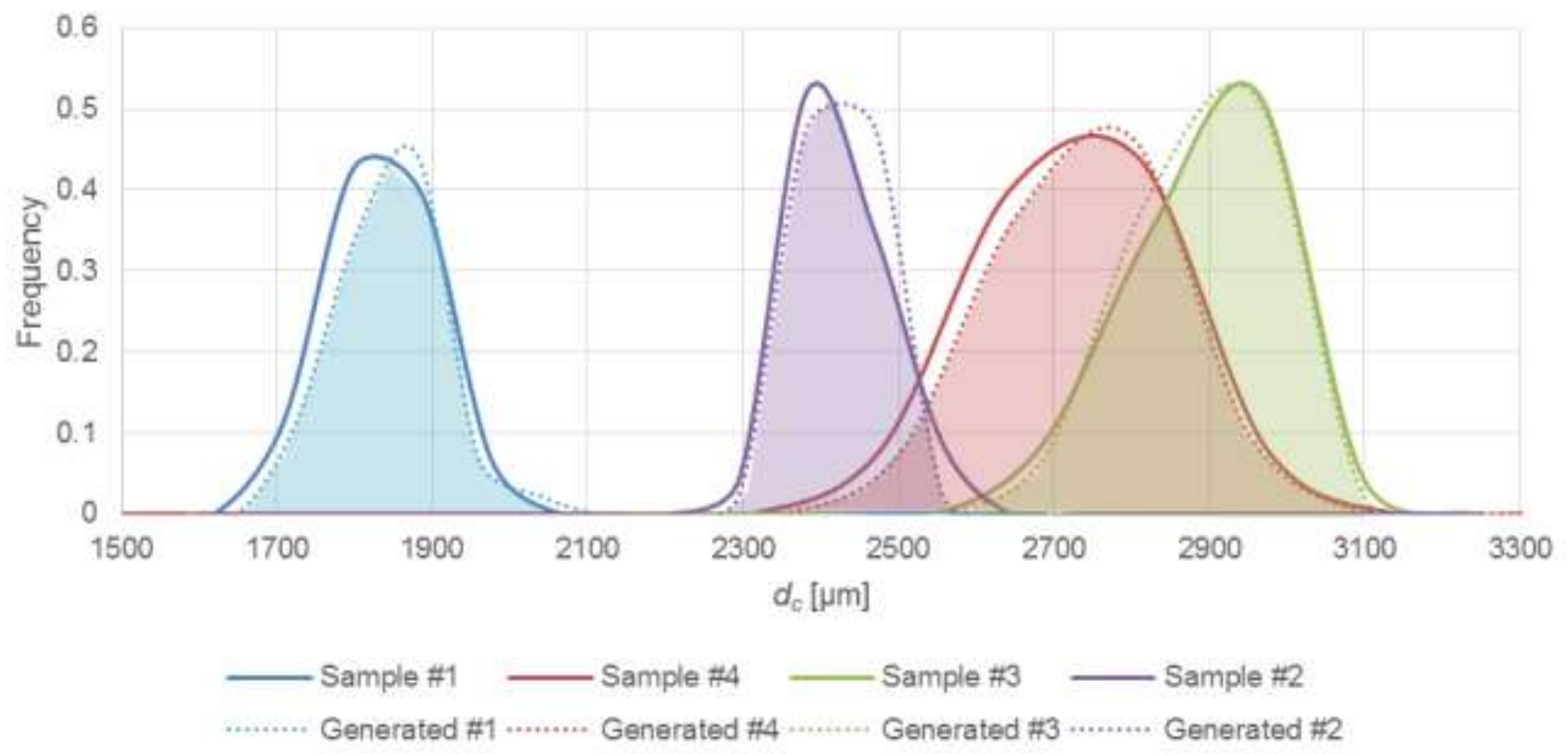

Fig. 9 - Equivalent cell diameter distributions of the 4 samples and respective cell size distribution for matched generated structures, as calculated by iMorph. Shared areas are filled. 


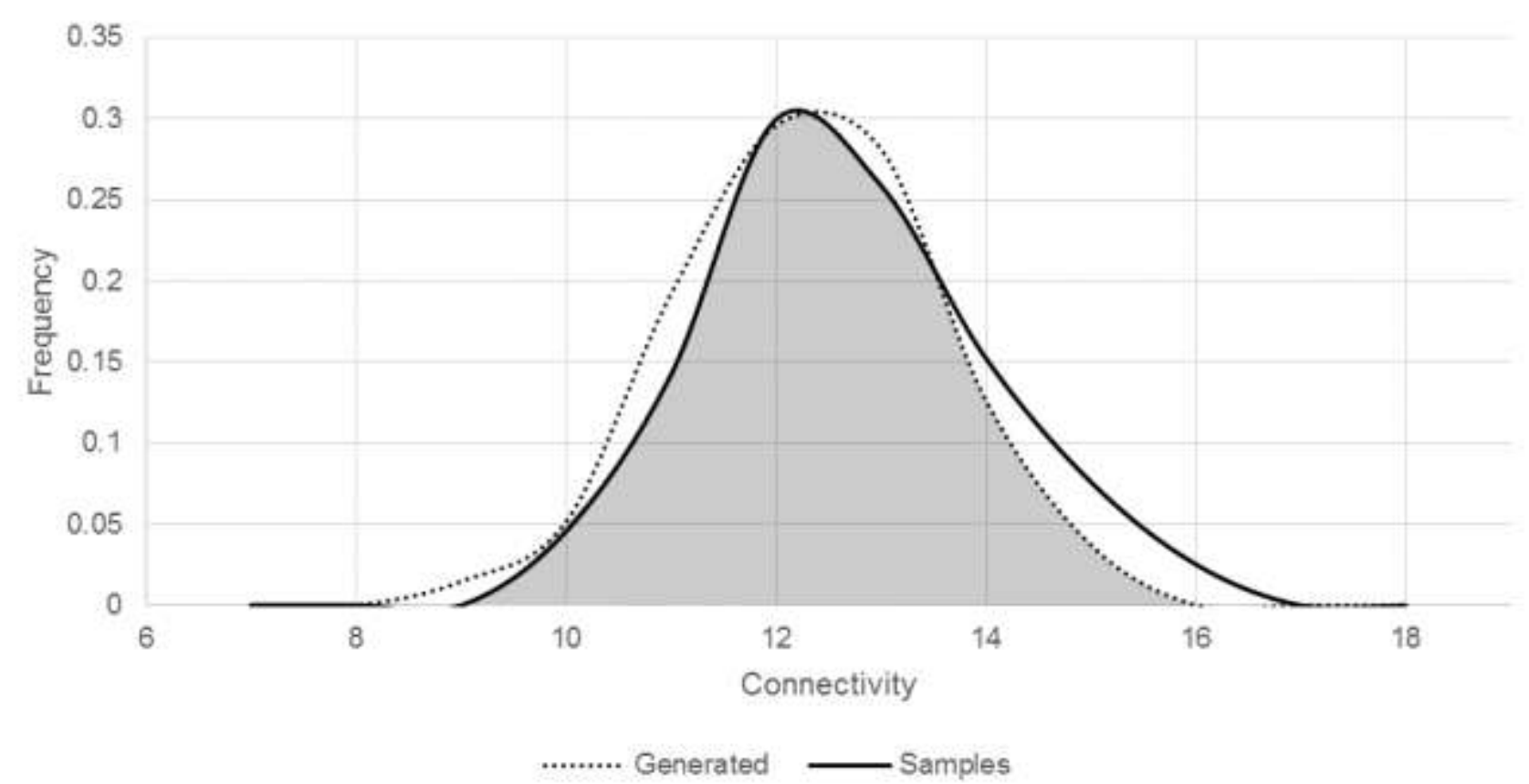

Fig. 10 - Average cell connectivity distribution of the 4 samples and average cell connectivity distribution for matched generated structures, as calculated by iMorph. Shared area is filled.

\subsection{Radiative properties calculation methodology}

Monte Carlo algorithms for calculation of radiative properties transfer have been extensively investigated in literature. The basic premise of the method is casting a large number of rays inside a digitally represented structure, and applying statistical treatment to the histories of the rays to characterize the radiative properties of the structure. Namely, for a given structure Monte Carlo radiation methods allow to calculate the extinction coefficient $\beta$, the scattering coefficient $\sigma$ and scattering albedo $\omega=\sigma / \beta$, the scattering phase function $\Phi(\theta)$. A comprehensive review of Monte Carlo methods for calculation of radiative properties in cellular media can be found in [28].

In the present paper, radiation propagation is assumed to obey the laws of Geometric Optics. This approximation is considered acceptable for metal and ceramic foams, where the strut diameter is typically in excess of $100 \mu \mathrm{m}$. The solid phase is considered opaque. The rays are launched from 
random points in the void phase, and are considered extinct (by absorption or scattering) when they intercept the solid surface. Rays that traverse the bounding box without intersection with the solid are launched again from the opposite side of the bounding box, after randomization of position [28].

The extinction coefficient is calculated as the inverse of the mean free path length of the rays:

$$
\beta=\frac{1}{\frac{\sum_{n}^{N} p_{n}}{N}}=\frac{1}{p_{\text {avg }}}
$$

It is worthwhile noting that such a characterization of the radiative properties of the medium is only valid if extinction in the medium follows a quasi-Beer-Lambert law. For all the geometries processed in this work, coherency of numerically calculated extinction with a Beer-Lambert law has been verified with an error of less than $1 \%$ across the entire range of values. In Fig. 11, a cloud of points representing computed values of the extinction probability distribution function for the four tomographically acquired samples and a selection of six generated samples is plotted along with an exponential fit.

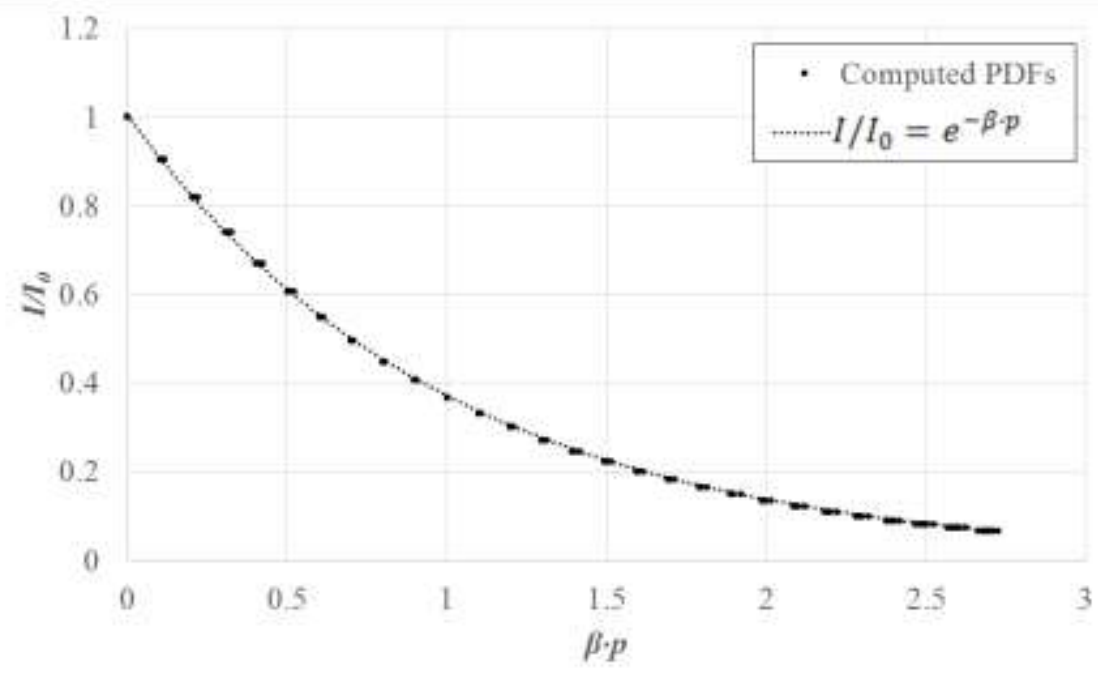

Fig. 11 - Computed extinction PDFs and exponential fit.

With respect to scattering, the reflection is assumed to be diffuse and, additionally, reflectivity is considered independent of incidence angle, as it is assumed in several previous works [22-29]. Under this assumption, the following relation holds for the determination of the scattering albedo:

$$
\omega=\rho_{s}
$$


Where $\rho_{s}$ is the surface reflectivity of the solid. Additionally, while Monte Carlo methods allow to numerically calculate the scattering phase function, it has been shown [24] that under the assumption of diffuse reflection, the numerically calculated scattering phase function for open cell foam structure closely matches the scattering phase function for opaque large spheres or randomly oriented convex opaque particles [11-13]:

$$
\Phi(\theta)=\frac{8}{3 \pi}(\sin \theta-\theta \cos \theta)
$$

This has been found to be true with respect to the geometries processed in this work. The computed value differ no more than $10 \%$ from the analytical function (3). In addition, the computed phase function asymmetry factor, $g$, ranges in an interval $-0.451 \leq g \leq-0.469$, within $6 \%$ from the value $g=-$ 0.444 that can be determined from (3). In Fig. 12, a cloud of points representing computed values of the scattering phase function for the four tomographically acquired samples and a selection of eight generated samples is plotted along with function (3).

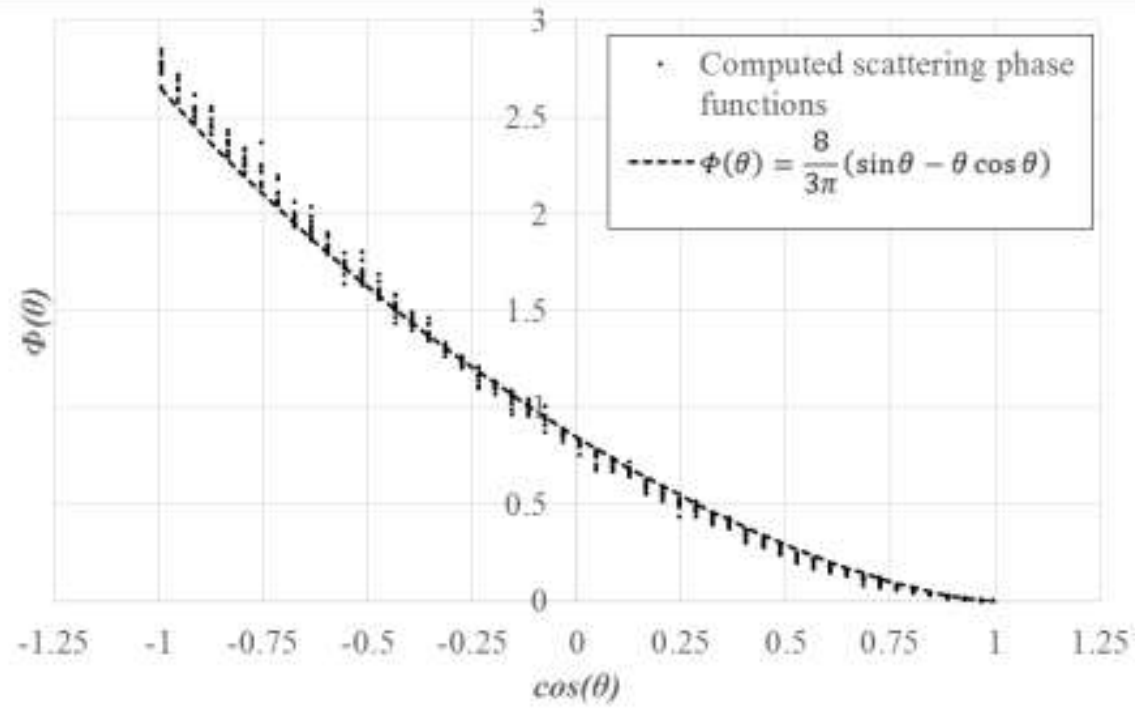

Fig. 12 - Computed scattering phase functions and analytical relation (3). 
For these reasons, the following analysis focuses on the extinction coefficient, $\beta$.

Furthermore, the foams have been considered isotropic in the following analysis. Indeed, anisotropy effects have usually been found to be small in metal foams, with directional values within $10 \%$ of average values [25, 41]. Furthermore, recent studies on aluminum foams have found anisotropy to be decreasing with increasing PPIs and practically undetectable in foams over 30 PPI [42] such as our samples.

The computations in the following have been executed on grids comprising a number of triangular mesh elements between $6 \times 10^{5}$ and $10^{6}$, with a memory occupation of $25-50 \mathrm{MB}$ per grid. Each computation involved the tracing of $10^{6}$ rays, with an average running time of around 22 minutes on 2.70 Ghz dual core processor.

\section{$3 \quad$ Results and discussion}

Four parameters and their influence have been analyzed in this study: dispersion of cell size distribution $(C V=\{0 \% ; 2.5 \% ; 5 \% ; 7.5 \% ; 10 \% ; 15 \%\})$, variation of strut diameter along the length $(t=$ $\{0.33 ; 0.5 ; 0.66 ; 0.84 ; 1.0\})$, porosity $(\varepsilon=\{98 \% ; 96 \%, 94 \% ; 92 \% ; 90 \% ; 88 \%\})$ and normalized curvature of strut cross section $(k=\{-0.29 ; 0 ; 0.5 ; 1.0\})$. A base case $(C V=5 \% ; t=1.0 ; \varepsilon=94 \% ; k=$ 1.0) is generated and then parameters are varied individually to evaluate the effects. The values of parameters have been chosen to span the range of typical values observed in real foams. All the results are computed for an average cell size of $1 \mathrm{~mm}$. It is worth noting that, operating under geometric optics approximation, the average mean free path is directly proportional to the average cell diameter, i.e. $\beta=$ $1 / p_{\text {avg }} \propto 1 / d_{c}$.

Our results show that not all parameters considered have comparable influence on radiation. 


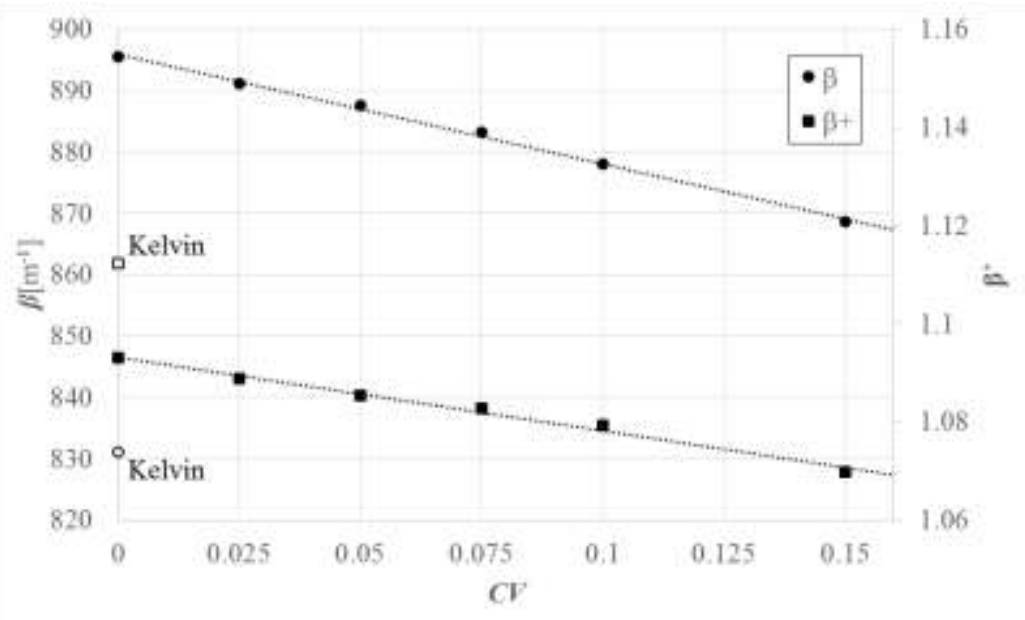

Fig. 13 - Effects of the variations of $C V$ on $\beta$ and $\beta^{+}$

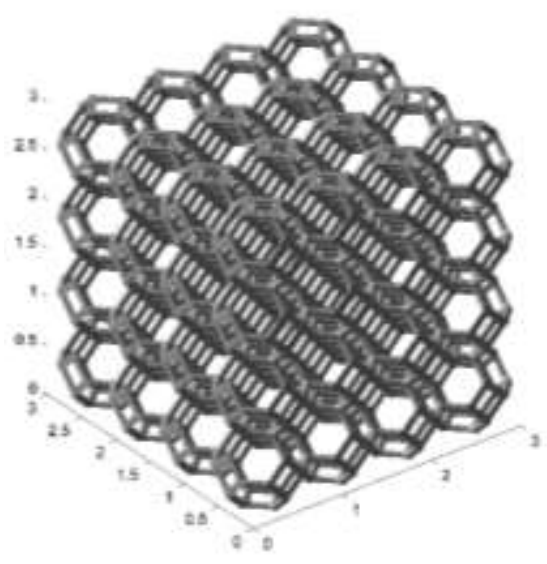

Fig. 14 - Kelvin foam

Dispersion of cell size does not appear to affect the extinction coefficient and the normalized extinction coefficient greatly. In fact, all structures considered appear to predict a small interval spanning $\pm 1.5 \%$ around the average value [Fig. 13].

The small influence of cell size dispersion prompts further investigation. Considering that a number of models in literature, both analytical [43] and numerical $[32,33]$ have used fully regular structures as models to compute the properties of real structures, it is deemed worth of interest comparing a structure of this type to the irregular ones. Thus, an additional structure has been generated using a regular Voronoi partition based on a BCC lattice (Kelvin foam) [Fig. 14].

While this single data point is further away from the average, the differences remain small in absolute value: the regular structures show higher normalized extinction coefficient $(+2 \%)$ and a lower extinction coefficient (-6\%) than random structures. This can be justified considering that struts of the regular structure meet at tetrahedral angles, thus minimizing self-shadowing and increasing the former, while on the other hand the regular structure allows the surface to be minimized, thus reducing the latter. It must be noted that long-range ordering effects in regular structures, namely the existence of preferential directions that never meet extinction, are ignored due to the nature of the Monte Carlo algorithms used, due to the choice of randomization of rays exiting the bounding box. This result 
partially supports the practice of using fully regular structures to estimate radiative properties in cellular media while minimizing required computational power, if some caution is used to correct the small errors incurred. However, the difference is such that the Kelvin structure has been excluded from the following analysis.

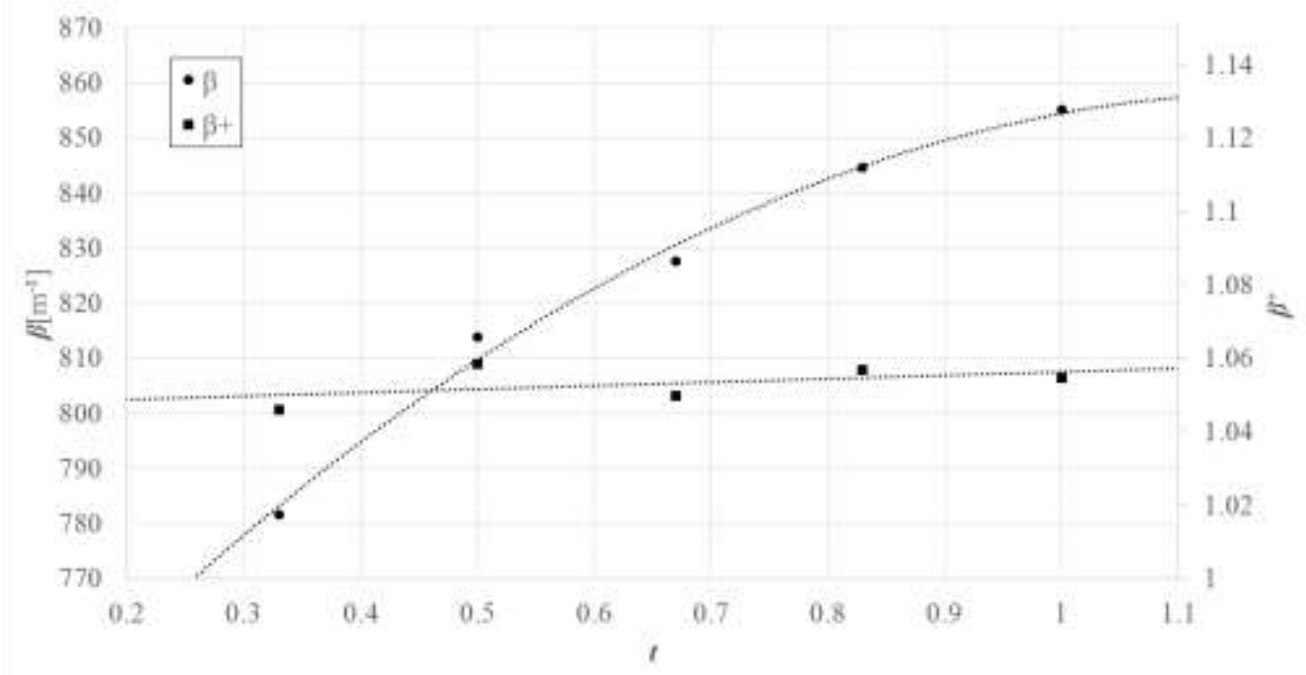

Fig. 15 - Effects of the variations of $t$ on $\beta$ and $\beta^{+}$

Variation of strut diameter along the length has moderate effects on the extinction coefficient. All values considered lie in a $\pm 5 \%$ interval. In addition, effects on the normalized extinction coefficient are minimal [Fig. 15]. This indicates that this parameter can be ignored if the specific surface area is already known through other measures.

In agreement with previous studies, porosity is found to have large effects on both the extinction coefficient and the normalized extinction coefficient. It is in fact by far the single parameter with the greatest influence among those considered [Fig. 16]. 


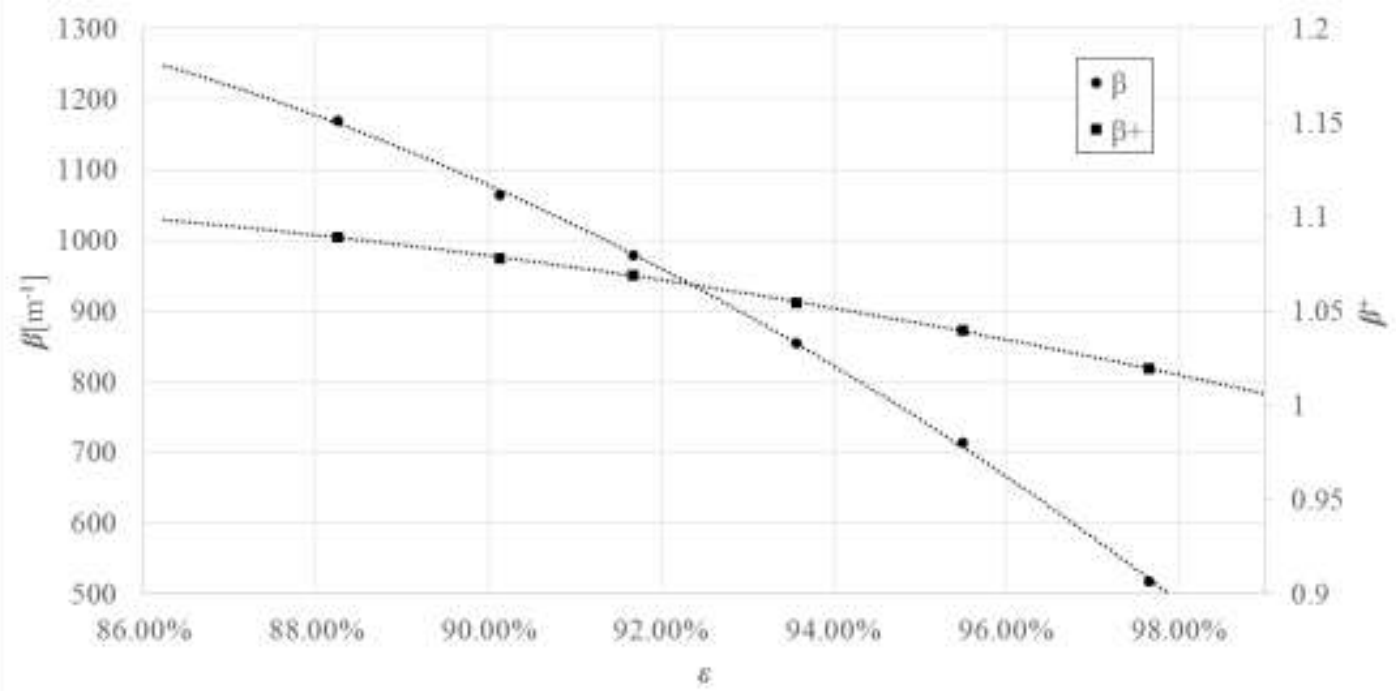

Fig. $16-$ Effects of the variations of $\varepsilon$ on $\beta$ and $\beta^{+}$

Strut cross section shape is also found to have large effects on the extinction coefficient and smaller, but not negligible effects on the normalized extinction coefficient. Overall, it is the second most influent parameter among those considered [Fig. 17].

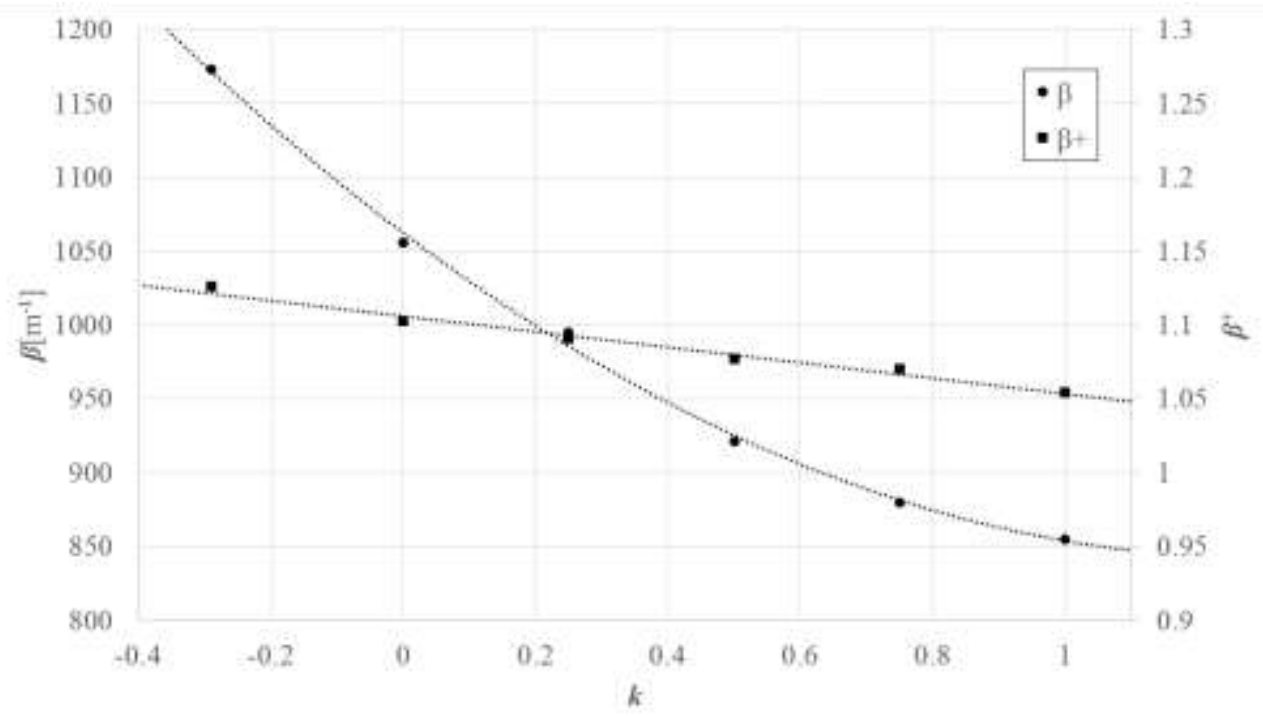

Fig. 17 - Effects of the variations of $k$ on $\beta$ and $\beta^{+}$ 


\section{Simplified analytical relations}

\subsection{Analytical relations - Presentation}

Based on the above findings, some simplified relations can be devised to calculate the extinction coefficient using a reduced set of parameters.

It can be noted that the normalized extinction eofficient, i.e. the ratio of extinction coefficient and specific surface area, varies in a quite restricted range and is sensitive only to porosity and strut cross section shape. Thus, one can devise a very precise estimate of the extinction coefficient using porosity ( $)$ [Fig. 16] and strut cross section shape (k) [Fig. 17] to estimate the normalized extinction coefficient with a small error, then multiplying by the specific surface area to obtain the value of $\beta$. Using our data, the following equation was estimated:

$$
\beta=\frac{S_{v}}{4 \varepsilon^{1.76[1+0.4(1-k)]}}
$$

The relation is proposed in a form similar to that of Brewster's relations for spherical beds [16]. The analytical solution for the independent scattering $\beta=S_{v} / 4$ is empirically adjusted with a factor depending from porosity and strut cross section shape

Physical consistency is assured, as $\lim _{\varepsilon \rightarrow 1} \beta=S_{v} / 4$. The strut cross section shape appears as a $(1-k)$ factor that can be thought of as "deviation from circular shape". The proposed relation fits all the data with a maximum error under 3\% and a root mean square error under 1\%. [Fig. 18] 


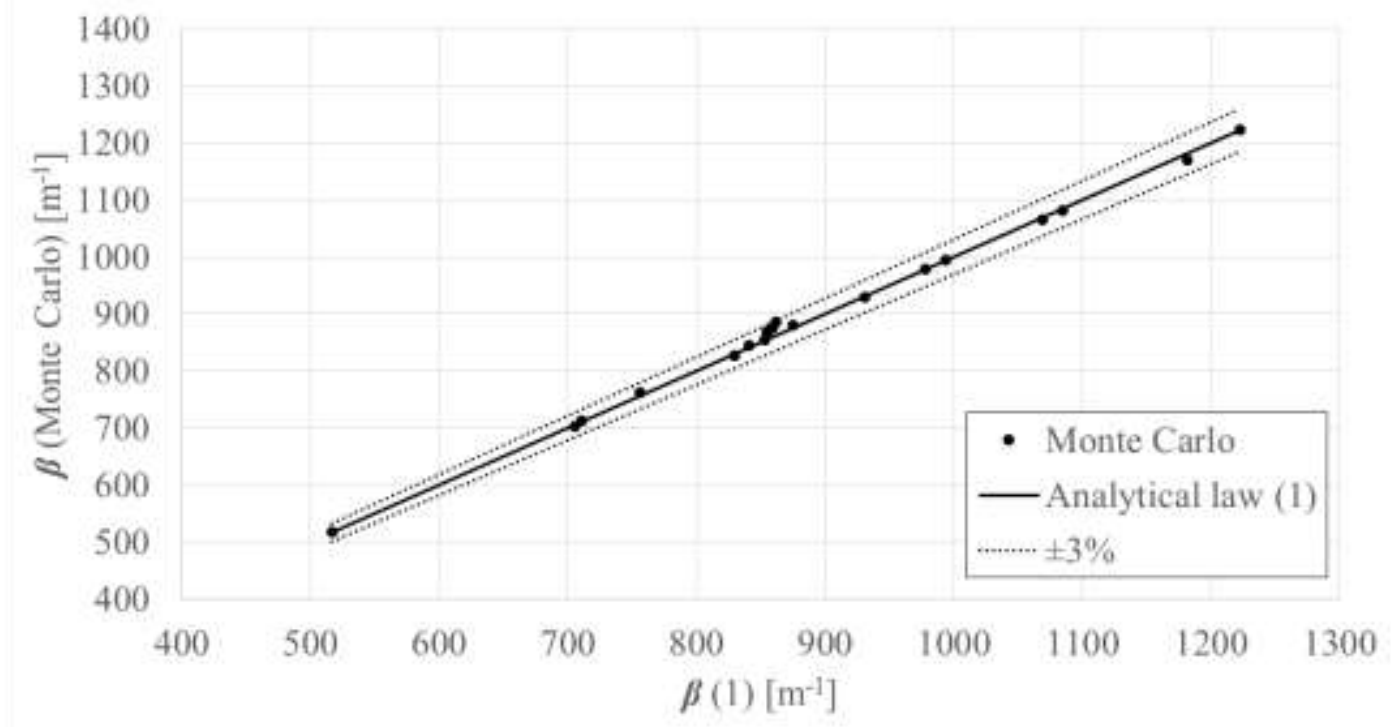

Fig. 18 - Comparison of analytical law (4) and Monte Carlo results on generated structures

Precise estimation of the specific surface area can be difficult to acquire. A relation that makes use of a more readily measured parameter may be of interest. The average equivalent diameter $d_{c}$ of the cell can be readily measured using simple imaging techniques. As seen previously [Fig. 15], variation of strut diameter along its length $(t)$ also affects the value of the extinction coefficient, when the specific surface area is unknown. Using the average equivalent diameter $d_{c}$, porosity $\varepsilon$, diameter ratio $t$ and normalized curvature $k$ as independent variables, the following equation was estimated:

$$
\beta=\frac{2.62 \sqrt{1-\varepsilon}\left[1+0.22(1-k)^{2}\right]\left[1-0.22(1-t)^{2}\right]}{d_{c}}
$$

The form of the relation has been chosen to generalize that typically seen in literature [39], adding dependence from strut cross section shape and variation of strut diameter along its length. Allowing the exponent associated with $(1-\varepsilon)$ to freely vary results in values very close to 0.5 , so the square root form has been retained and the exponent imposed in the regression. Strut cross section shape and variation of strut diameter along its length appear as $(1-k)$ and $(1-t)$ factors that can be thought of as "deviation from circular shape" and "deviation from constant diameter" respectively. The exponent of two associated with $(1-k)$ and $(1-t)$ is chosen a priori, following from the shape of the curve on Fig. 
13 and 15 . The proposed relation fits all the data with a maximum error under $2.5 \%$ and a root mean square error under 1\%. [Fig. 19]

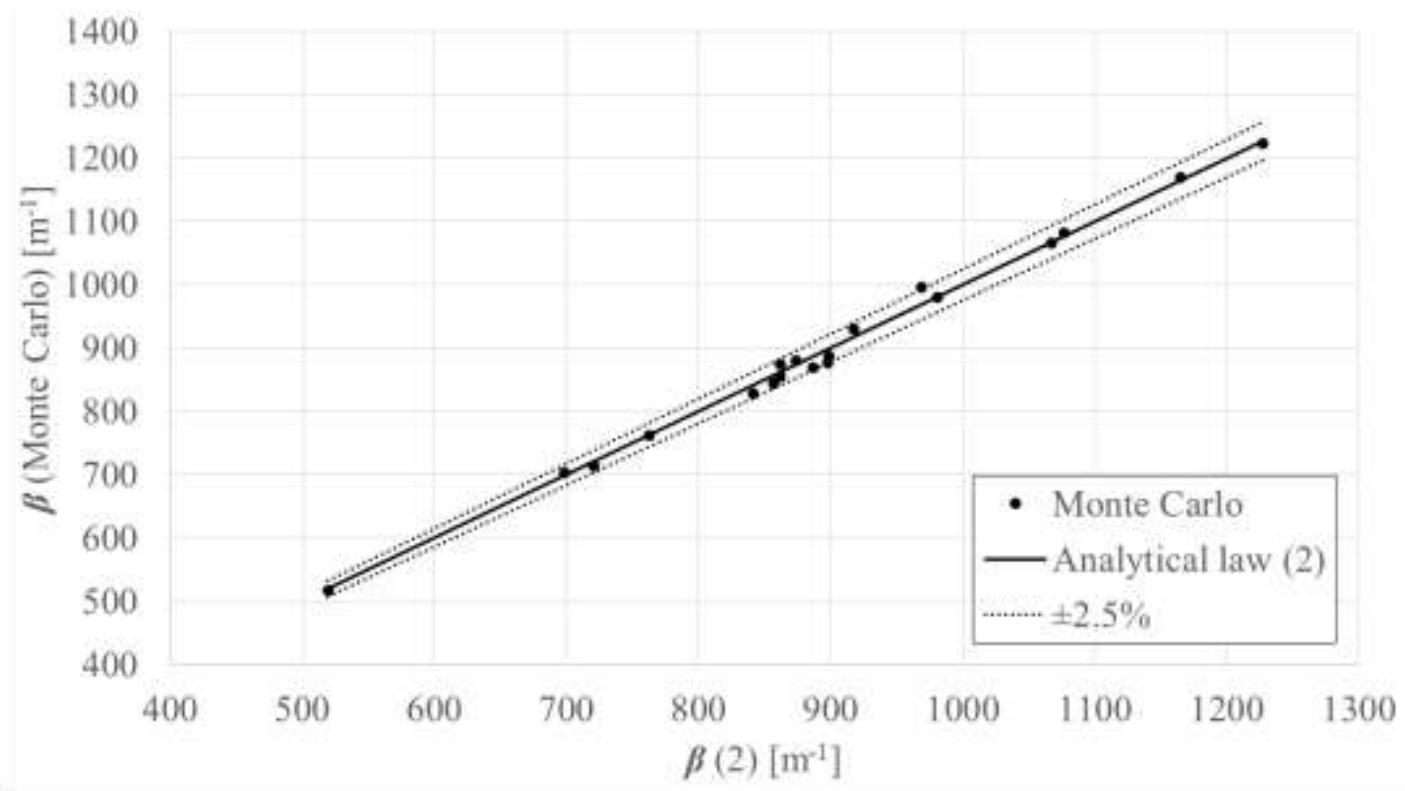

Fig. 19 - Comparison of analytical law (5) and Monte Carlo results on generated structures.

Dimensionless parameters $k$ and $t$, necessary for the computation of (4) and (5), can be easily determined by stereological techniques. In this work, iMorph was used as a stereological tool to devise relations between $k$ [Appendix A] and $t$ [Appendix B] and measurable stereological quantities.

\subsection{Analytical relations - Validation}

To validate the analytical relations (4) and (5), tomographic data from the same four samples presented in Section 2 have been used in direct Monte Carlo simulations, and the results have been compared with those obtained applying the analytical relations to morphological data from the same samples. The morphological data required as input have been obtained using iMorph and are listed in Table 2. For further reference, Fig. 20 also shows a typical tomography slice for each of the four samples. 


\begin{tabular}{|c|c|c|c|c|c|}
\hline $\begin{array}{c}\text { Sample } \\
\text { number }\end{array}$ & $\varepsilon$ & $d_{c}[\mathrm{um}]$ & $S_{v}\left[\mathrm{~m}^{-1}\right]$ & $\begin{array}{c}k \\
\text { (Appendix A) }\end{array}$ & $\begin{array}{c}t \\
\text { (Appendix B) }\end{array}$ \\
\hline 1 & $87.2 \%$ & 1842 & 1596 & 0.42 & 0.52 \\
\hline 2 & $89.3 \%$ & 2431 & 1066 & 0.57 & 0.4 \\
\hline 3 & $93.6 \%$ & 2892 & 742 & 0.36 & 0.34 \\
\hline 4 & $96.3 \%$ & 2725 & 756 & 0.16 & 0.53 \\
\hline
\end{tabular}

Tab. 2 - Measured morphological characteristics of the four samples.

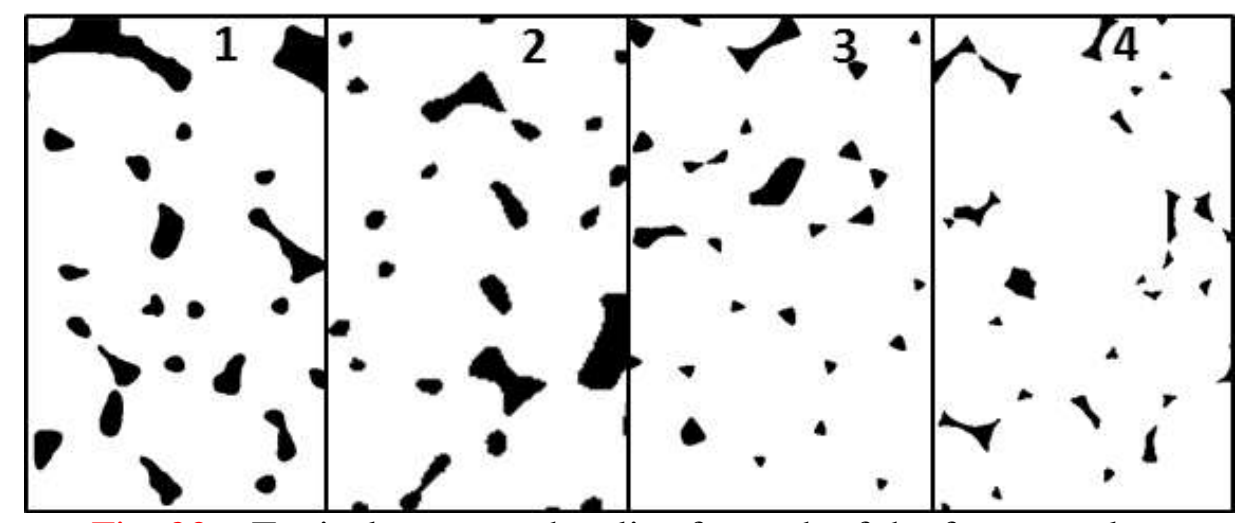

Fig. 20 - Typical tomography slice for each of the four samples.

The results of the validation can be seen in Table 3 .

\begin{tabular}{|c|c|c|c|c|c|}
\hline $\begin{array}{c}\text { Sample } \\
\text { number }\end{array}$ & $\begin{array}{c}\beta\left[\mathrm{m}^{-1}\right] \\
(\mathrm{MC})\end{array}$ & $\begin{array}{c}\beta\left[\mathrm{m}^{-1}\right] \\
(1)\end{array}$ & $\begin{array}{c}\text { Relative error } \\
(1)\end{array}$ & $\begin{array}{c}\beta\left[\mathrm{m}^{-1}\right] \\
(2)\end{array}$ & $\begin{array}{c}\text { Relative error } \\
(2)\end{array}$ \\
\hline 1 & 524.6 & 533.8 & $1.76 \%$ & 510.9 & $-2.61 \%$ \\
\hline 2 & 335.5 & 336.5 & $0.31 \%$ & 336.9 & $0.43 \%$ \\
\hline 3 & 217.8 & 214.6 & $-1.45 \%$ & 224.6 & $3.16 \%$ \\
\hline 4 & 205 & 206.6 & $0.77 \%$ & 203.1 & $-0.95 \%$ \\
\hline
\end{tabular}

Tab. 3 - Comparison of direct Monte Carlo computations on the four samples, and relations (3) and (4). Morphological data from Tab. 2.

As expected, relation (4) affords the best accuracy, with a maximum error below $2 \%$ and a root mean square error of $1.21 \%$. Relation (5), on the other hand, affords a maximum error under $3.5 \%$ and a root mean square error of $2.11 \%$. In the light of the wide variability of geometrical characteristics of the four samples considered, the agreement between analytical results and Monte Carlo simulations on tomographic data is considered very satisfying for both relations.

In addition, the accuracy of relations (1) and (2) has been compared with two reference relations from literature. The first is the one given by Brewster [16] for spherical beds and uses $S_{v}$ as a variable: 


$$
\beta=\frac{S_{v}}{4 \varepsilon}
$$

The second is the one given by Glicksman et al. [43] and uses the cell diameter as a variable:

$$
\beta=4.09 \frac{\sqrt{1-\varepsilon}}{d_{c, G}}
$$

Considering that the cell representation used in [43] is that of an equivalent dodecahedron and that the mean diameter of the dodecahedron $d_{c, G}$ is used, an equal volume rescaling from equivalent sphere to dodecahedron has been adopted for the equivalent cell diameter $\left(d_{c, G}=1.08 d_{c}[44]\right)$. Additionally, the calculations in [43] use a correction factor of $\sqrt{2 / 3}$ to account for the concavity of the strut cross section typically observed in plastic foams [45].

As the samples used in this study do present convex cross sections, this correction factor has been reversed. This ultimately yields:

$$
\beta=3.09 \frac{\sqrt{1-\varepsilon}}{d_{c}}
$$

The results of the comparison can be seen in Tab. 4 .

\begin{tabular}{|c|c|c|c|c|}
\hline & \multicolumn{2}{|c|}{$S_{v}$} & \multicolumn{2}{c|}{$d_{c}$} \\
\hline $\begin{array}{c}\text { Sample } \\
\text { number }\end{array}$ & Relative error (4) & $\begin{array}{c}\text { Relative error (6) } \\
{[16]}\end{array}$ & Relative error (5) & $\begin{array}{c}\text { Relative error (7b) } \\
{[41]}\end{array}$ \\
\hline 1 & $1.76 \%$ & $-12.82 \%$ & $-2.61 \%$ & $5.75 \%$ \\
\hline 2 & $0.31 \%$ & $-11.06 \%$ & $0.43 \%$ & $14.79 \%$ \\
\hline 3 & $-1.45 \%$ & $-9.00 \%$ & $3.16 \%$ & $14.66 \%$ \\
\hline 4 & $0.77 \%$ & $-4.25 \%$ & $-0.95 \%$ & $-1.37 \%$ \\
\hline \multicolumn{4}{|c|}{} \\
\hline Max error & $1.76 \%$ & $-12.82 \%$ & $3.16 \%$ & $14.79 \%$ \\
\hline RMS error & $1.21 \%$ & $9.82 \%$ & $2.11 \%$ & $10.82 \%$ \\
\hline
\end{tabular}

Tab. 4 - Comparison of relative error of relations (4), (5) to reference relations in the literature (6) and (7b). Morphological data from Tab. 2.

As can be seen in the table, all the proposed relations afford a significant accuracy improvement over the respective reference relations. It can also be noted that, while reference relations (6) and (7b) 
consistently under predict and tendentially over predict (respectively) the real value of the extinction coefficient, errors for the proposed relations (4) and (5) appear to be randomly distributed.

Finally, it is interesting to propose simplified forms for equations (4) and (5), i.e. forms that include less dimensionless parameters. Optimal values for normalized curvature $k$ and diameter ratio $t$ are determined by averaging the values measured for the four samples. Setting $k=0.4$, from equation (4) we obtain:

$$
\beta=\frac{S_{v}}{4 \varepsilon^{2.18}}
$$

Setting $t=0.45$, from equation (5) we obtain:

$$
\beta=\frac{2.45 \sqrt{1-\varepsilon}\left[1+0.22(1-k)^{2}\right]}{d_{c}}
$$

Then setting $k=0.4$ we obtain:

$$
\beta=\frac{2.64 \sqrt{1-\varepsilon}}{d_{c}}
$$

\begin{tabular}{|c|c|c|c|c|c|c|c|}
\hline & \multicolumn{3}{|c|}{$S_{v}$} & \multicolumn{4}{|c|}{$d_{c}$} \\
\hline $\begin{array}{l}\text { Sample } \\
\text { number }\end{array}$ & $\begin{array}{l}\text { Relative } \\
\text { error (4) }\end{array}$ & $\begin{array}{l}\text { Relative } \\
\text { error (8) }\end{array}$ & $\begin{array}{c}\text { Relative } \\
\text { error (6) } \\
{[16]}\end{array}$ & $\begin{array}{l}\text { Relative } \\
\text { error (5) }\end{array}$ & $\begin{array}{l}\text { Relative } \\
\text { error (9) }\end{array}$ & $\begin{array}{l}\text { Relative } \\
\text { error (10) }\end{array}$ & $\begin{array}{c}\text { Relative } \\
\text { error }(7 b) \\
{[43]}\end{array}$ \\
\hline 1 & $1.76 \%$ & $2.42 \%$ & $-12.82 \%$ & $-2.61 \%$ & $-3.85 \%$ & $-2.42 \%$ & $5.75 \%$ \\
\hline 2 & $0.31 \%$ & $1.65 \%$ & $-11.06 \%$ & $0.43 \%$ & $2.29 \%$ & $5.92 \%$ & $14.79 \%$ \\
\hline 3 & $-1.45 \%$ & $-1.65 \%$ & $-9.00 \%$ & $3.16 \%$ & $7.03 \%$ & $5.80 \%$ & $14.66 \%$ \\
\hline 4 & $0.77 \%$ & $0.12 \%$ & $-4.25 \%$ & $-0.95 \%$ & $-2.43 \%$ & $-8.99 \%$ & $-1.37 \%$ \\
\hline Max error & $1.76 \%$ & $2.42 \%$ & $-12.82 \%$ & $3.16 \%$ & $7.03 \%$ & $-8.99 \%$ & $14.79 \%$ \\
\hline RMS error & $1.21 \%$ & $1.68 \%$ & $9.82 \%$ & $2.11 \%$ & $4.34 \%$ & $6.23 \%$ & $10.82 \%$ \\
\hline
\end{tabular}

In Tab. 5, the accuracy of relations (8)-(10) is reported, once again in comparison with original relations (4)-(5) and reference relations (6)-(7b).

Tab. 5 - Comparison of relative error of relations (8)-(10) in comparison with original relations (4) and (5) and reference relations (6) and (7b). Morphological data from Tab. 2. 
As expected, decreasing the number of dimensionless parameters decreases the accuracy. Therefore, RMS error for relation (8) is 50\% larger than RMS error for relation (4), and RMS error for relations (9) and (10) is respectively $100 \%$ and $200 \%$ larger than RMS error for relation (5). However, relations (8) and (9)-(10) still offer a significant accuracy improvement over relations (6) and (7b) respectively. As previously, it can also be noted that errors for proposed relations (8)-(10) appear to be randomly distributed, as opposed to asymmetrical errors from relations (6) and (7b).

\section{Conclusions}

In this study, a novel methodology to generate open cell structures along with Monte Carlo numerical methods have used to predict radiative properties of open cell solid foams. Triangular meshes were used to replicate real foams, with finely controlled cell size distribution and strut shape, and smooth, realistic strut intersections free of self-intersecting elements. To demonstrate the capabilities of the algorithm, the geometrical characteristics of the resulting structures have been compared to those of four real foam samples, with satisfying results.

The methodology was subsequently applied, individually varying four parameters (porosity, strut cross section, strut minimum to maximum diameter ratio, dispersion of cell size distribution) in the typical experimentally observed range, to produce a number of structures.

The application of Monte Carlo ray tracing algorithms to the resulting structures made it possible to reduce the number of parameters and to devise and propose two analytical relations to determine the value of the extinction coefficient, fitting all the computed data with small error.

The relations have been validated by comparison with results from direct Monte Carlo computations on four real foam samples with satisfying results, and then compared to existing reference relations from 
literature. Good absolute accuracy and significant accuracy improvement over existing relations is achieved.

Additionally, simplified forms of the proposed relations have been obtained and their accuracy compared with full form relations and with reference relations. The simplified relations are less accurate than the original ones, but they still represent a significant improvement over reference relations. These relations should be useful for the design of materials e.g. for energy efficiency. 


\section{Appendix A - Evaluating the normalized curvature $(k)$}

To evaluate the normalized curvature $k$, it is necessary to establish a relation between $k$ and some stereological measures. In this work, iMorph [40] was used as stereological tool, so in the following a relation is proposed to derive the normalized curvature from measures provided by the software.

The cross-section measurement function available in iMorph has been used. The function automatically identifies struts and then provides geometrical measures for the identified 2D cross sections, namely surface $S_{s}$, perimeter $P_{s}$, diameter of inscribed circle $d_{i}$, diameter of circumscribed circle $d_{o}$.

A relation between the normalized curvature and a dimensionless ratio of the measures obtained by the software has been established.

To this end, three generated geometries at set values of $k=\{-0.29,0.35,1\}$ have been treated.

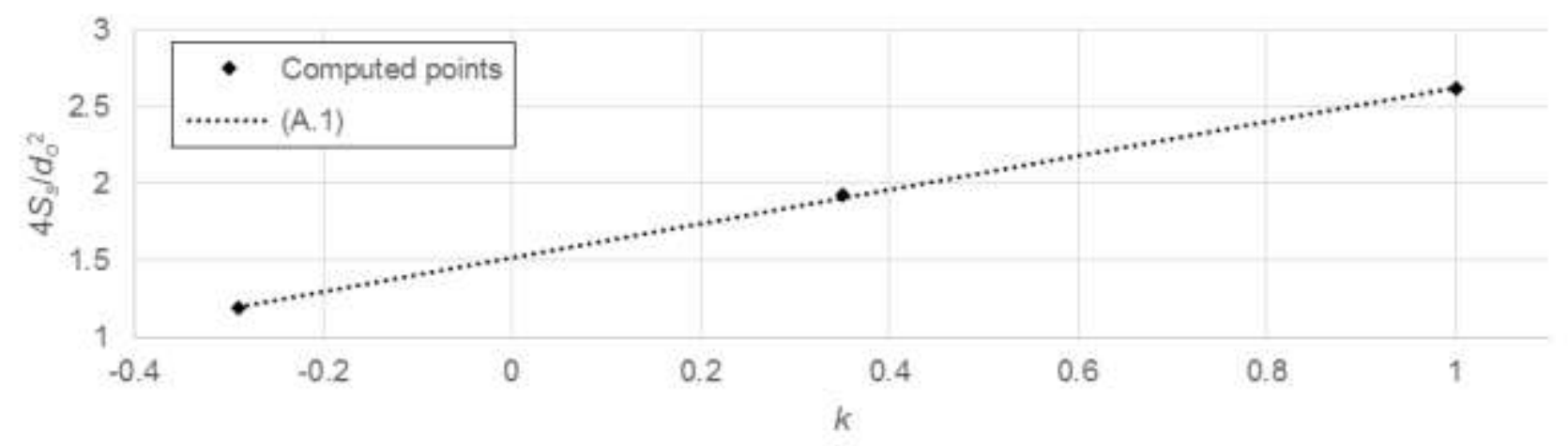

Fig. A.1 - Variation of $4 S_{s} / d_{o}^{2}$ with $k$.

The dimensionless ratio $4 S_{s} / d_{o}^{2}$ shows a linear relation with $\mathrm{k}$ [Fig. A.1], which can be written as:

$$
4 S_{s} /_{d_{o}^{2}}=1.11 k+1.52
$$


In addition, this ratio can be easily related to the ratio of the area of the cross section to the area of a simple planar shape enclosing the cross section. With reference to the area of the circumscribed circle of the cross section [46]:

$$
\pi^{S_{s}} /_{A_{\text {circle }}}=1.11 k+1.52
$$

With reference to the area of an equilateral triangle having the same vertices as the cross section [45]:

$$
\frac{3 \sqrt{3}}{4} S_{s} / A_{\text {triangle }}=1.11 k+1.52
$$

It must be stressed that, while relations (A.1)-(A.3) have been obtained using iMorph, they can be in principle applied to the same geometrical parameters measured using any stereological technique.

Finally, relation (A.1) was used as reference to attribute values of normalized curvature to the tomographic samples [Fig. A.2].

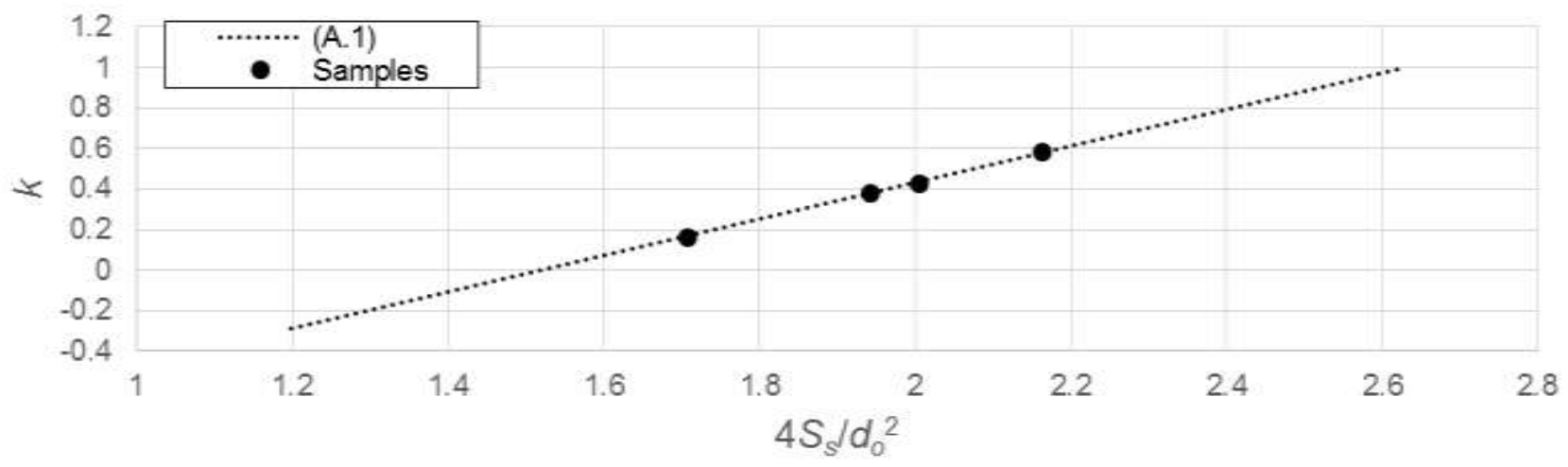

Fig. A.2 - Determination of $k$ for the four samples. 


\section{Appendix B - Evaluating the diameter ratio $(t)$}

To evaluate the diameter ratio $t$, it is necessary to establish a relation between $t$ and some stereological measures. It is worth noting that the diameter ratio can also be readily measured from SEM scans of the foam [19], but in this work, iMorph [40] was used as stereological tool, so in the following a relation is proposed to derive the diameter ratio from measures provided by the software.

The cross-section measurement function available in iMorph has been used, together with a granulometry and segmentation of the solid phase, which allowed identification and measurement of strut junctions. The relevant geometrical measures provided are:

- For the strut cross sections, the diameter of inscribed circle $d_{i}$ and the diameter of circumscribed circle $d_{o}$.

- For the strut junctions, the average equivalent diameter $d_{j, e q}$ and the maximum diameter $d_{j, \max }$.

A relation between the diameter ratio and a dimensionless ratio of the measures obtained by the software has been established. The main concern is stability of the measure with respect to variation of other geometrical parameters, namely porosity and strut cross section shape. Four geometries, with porosity and cross section shapes corresponding to those measured for the four samples, have been generated with a constant $t=0.5$. Ideally, one would be able to find a ratio that stays constant over the four geometries.

\begin{tabular}{|c|c|c|c|c|}
\hline $\begin{array}{c}\text { Reference sample } \\
\text { number }\end{array}$ & $\varepsilon$ & $k$ & $t$ & $\frac{d_{o}+d_{i}}{2 d_{j, \max }}$ \\
\hline 1 & $87.2 \%$ & 0.42 & 0.5 & 0.633 \\
\hline 2 & $89.3 \%$ & 0.57 & 0.5 & 0.633 \\
\hline 3 & $93.6 \%$ & 0.36 & 0.5 & 0.645 \\
\hline 4 & $96.3 \%$ & 0.16 & 0.5 & 0.642 \\
\hline
\end{tabular}

Tab. B.1 - Sensitivity of $\left(d_{o}+d_{i}\right) /\left(2 d_{j, \max }\right)$ to variations of $\varepsilon$ and $k$ for a constant $\mathrm{t}=0.5$. 
The dimensionless ratio $\left(d_{o}+d_{i}\right) /\left(2 d_{j, \max }\right)$ has been picked. It shows very low sensitivity to variations of porosity and strut cross section shape, with all values falling in a $\pm 1 \%$ interval. [Tab. B.1]. To relate this ratio to the value of the diameter ratio, three geometries at set values of $t=\{0.2,0.5,1\}$ have been treated, and the dimensionless ratio has been plotted as a function of $t$ [Fig. B.1].

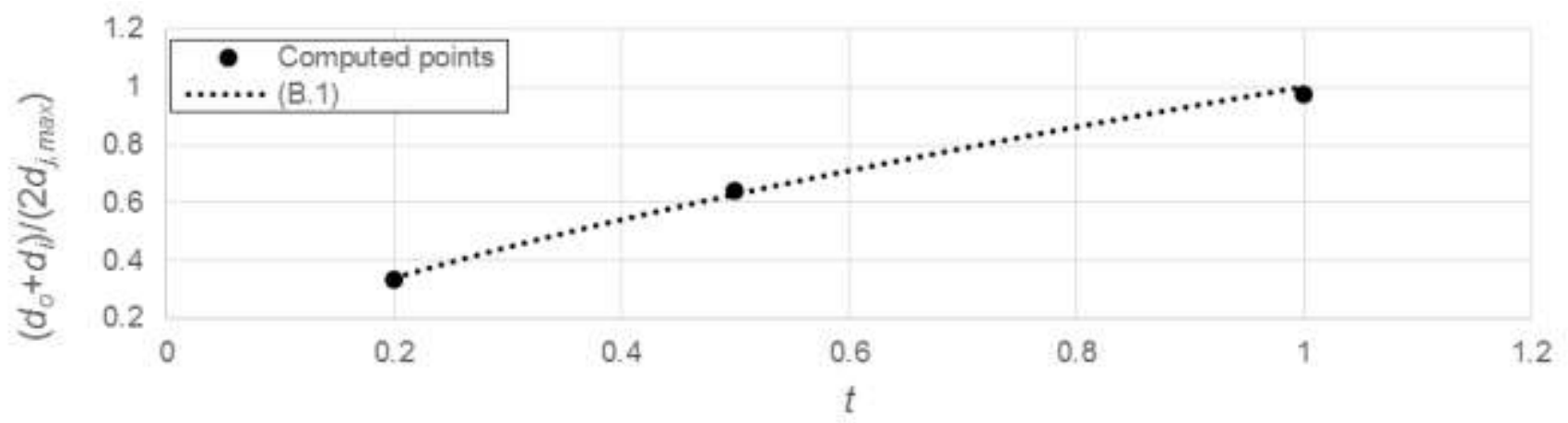

Fig. B. 1 -Variation of $\left(d_{o}+d_{i}\right) /\left(2 d_{j, \max }\right)$ with $t$.

The relationship is not linear, but a simple power law (plotted in the figure) fits the data points very well:

$$
\frac{d_{o}+d_{i}}{2 d_{j, \max }}=t^{\frac{2}{3}}
$$

It must be stressed that, while relation (9) has been obtained using iMorph, it can be in principle applied to the same geometrical parameters measured using any stereological technique.

Finally, relation (9) is used as reference to determine the value of the diameter ratio for the four samples [Fig. B.2]. 


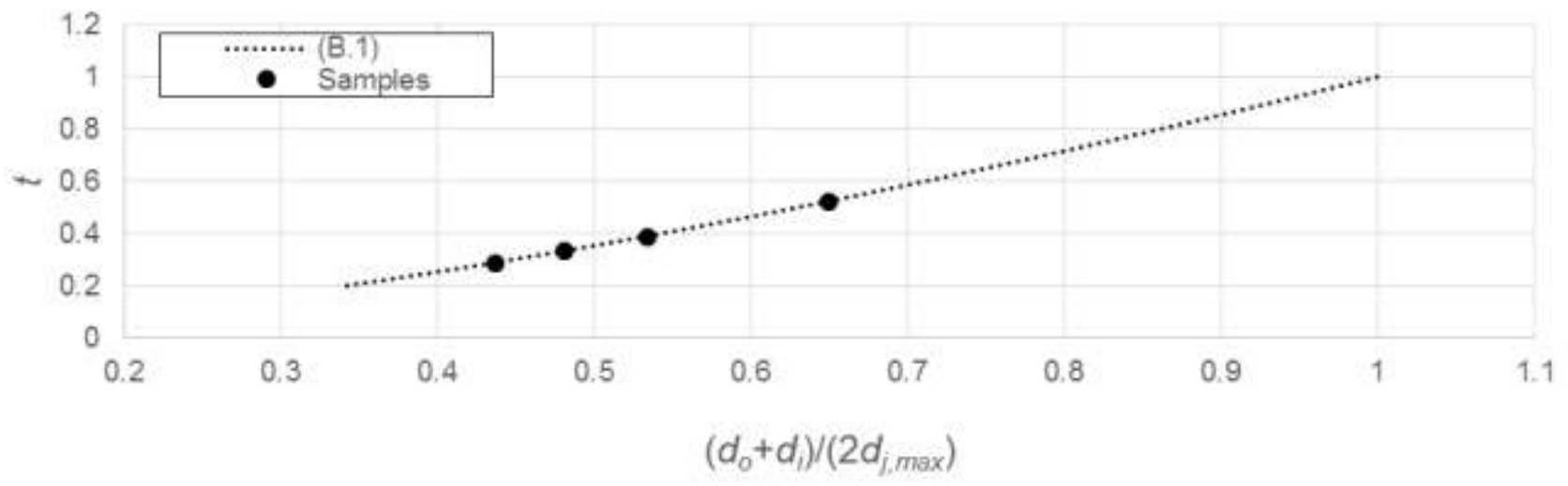

Fig. B.2 - Determination of $t$ for the four samples. 


\section{Acknowledgements}

Salvatore Cunsolo holds a doctoral fellowship from Région Rhône Alpes.

W.K.S. Chiu acknowledge financial support from an Energy Frontier Research Center on Science

Based Nano-Structure Design and Synthesis of Heterogeneous Functional Materials for Energy

Systems (HeteroFoaM Center) funded by the U.S. Department of Energy, Office of Science, Office of

Basic Energy Sciences (Award DE-SC0001061).

\section{References}

[1] Collishaw, P. G., \& Evans, J. R. G. (1994). An assessment of expressions for the apparent thermal conductivity of cellular materials. Journal of materials science, 29(9), 2261-2273.

[2] Placido, E., Arduini-Schuster, M. C., \& Kuhn, J. (2005). Thermal properties predictive model for insulating foams. Infrared physics \& technology, 46(3), 219-231.

[3] Coquard, R., \& Baillis, D. (2006). Modeling of heat transfer in low-density EPS foams. Journal of heat transfer, 128(6), 538-549.

[4] Kaemmerlen, A., Vo, C., Asllanaj, F., Jeandel, G., \& Baillis, D. (2010). Radiative properties of extruded polystyrene foams: Predictive model and experimental results. Journal of Quantitative Spectroscopy and Radiative Transfer, 111(6), 865-877.

[5] Fend, T., Reutter, O., Bauer, J., \& Hoffschmidt, B. (2004). Two novel high-porosity materials as volumetric receivers for concentrated solar radiation. Solar energy materials and solar cells, 84(1), 291-304.

[6] Lu, T. J., Stone, H. A., \& Ashby, M. F. (1998). Heat transfer in open-cell metal foams. Acta Materialia, 46(10), 3619-3635.

[7] Gauthier, S., Lebas, E., \& Baillis, D. (2007). SFGP 2007-natural gas/hydrogen mixture combustion in a porous radiant burner. International Journal of Chemical Reactor Engineering, $5(1)$.

[8] Gauthier, S., Nicolle, A., \& Baillis, D. (2008). Investigation of the flame structure and nitrogen oxides formation in lean porous premixed combustion of natural gas/hydrogen blends. International Journal of Hydrogen Energy, 33(18), 4893-4905.

[9] Lu, T. J., \& Chen, C. (1999). Thermal transport and fire retardance properties of cellular aluminium alloys. Acta Materialia, 47(5), 1469-1485.

[10] Mendes, M.A., Goetze, P., Talukdar, P., Werzner, E., Demuth, C., Rössger, P., Wulf, R., Gross, U., Trimis, D. and Ray, S. (2016). Measurement and simplified numerical prediction of effective thermal conductivity of open-cell ceramic foams at high temperature. International Journal of Heat and Mass Transfer, 102, pp.396-406.

[11] Siegel, R., \& Howell, J. R. (1992). Thermal radiation heat transfer. National Aeronautics and Space Administration, Cleveland, $\mathrm{OH}$ (United States). Lewis Research Center.

[12] Modest, M. F. (2013). Radiative heat transfer. Academic press.

[13] Brewster, M. Q. (1992). Thermal radiative transfer and properties. John Wiley \& Sons. 
[14] Dombrovsky, L. A., \& Baillis, D. (2010). Thermal radiation in disperse systems: An engineering approach. New York: Begell House.

[15] Randrianalisoa, J., \& Baillis, D. (2010). Radiative properties of densely packed spheres in semitransparent media: A new geometric optics approach. Journal of Quantitative Spectroscopy and Radiative Transfer, 111(10), 1372-1388.

[16] Brewster, Q. (2004). Volume scattering of radiation in packed beds of large, opaque spheres. Journal of heat transfer, 126(6), 1048-1050.

[17] Kamiuto, K. (1990). Correlated radiative transfer in packed-sphere systems. Journal of Quantitative Spectroscopy and Radiative Transfer, 43(1), 39-43.

[18] Baillis, D., Coquard, R., Randrianalisoa, J. H., Dombrovsky, L. A., \& Viskanta, R. (2013). Thermal radiation properties of highly porous cellular foams. Special Topics \& Reviews in Porous Media: An International Journal, 4(2).

[19] Baillis, D., \& Sacadura, J. F. (2000). Thermal radiation properties of dispersed media: theoretical prediction and experimental characterization. Journal of Quantitative Spectroscopy and Radiative Transfer, 67(5), 327-363.

[20] Patel, V. M., \& Talukdar, P. (2016). Evaluation of radiative properties of a representative foam structure using blocked-off region approach integrated with finite volume method. International Journal of Thermal Sciences, 108, 89-99.

[21] Tancrez, M., \& Taine, J. (2004). Direct identification of absorption and scattering coefficients and phase function of a porous medium by a Monte Carlo technique. International Journal of Heat and Mass Transfer, 47(2), 373-383.

[22] Zeghondy, B., Iacona, E., \& Taine, J. (2006). Determination of the anisotropic radiative properties of a porous material by radiative distribution function identification (RDFI). International Journal of Heat and Mass Transfer, 49(17), 2810-2819.

[23] Zeghondy, B., Iacona, E., \& Taine, J. (2006). Experimental and RDFI calculated radiative properties of a mullite foam. International Journal of Heat and Mass Transfer, 49(19), 3702-3707.

[24] Petrasch, J., Wyss, P., \& Steinfeld, A. (2007). Tomography-based Monte Carlo determination of radiative properties of reticulate porous ceramics. Journal of Quantitative Spectroscopy and Radiative Transfer, 105(2), 180-197.

[25] Coquard, R., Rousseau, B., Echegut, P., Baillis, D., Gomart, H., \& Iacona, E. (2012). Investigations of the radiative properties of $\mathrm{Al}-\mathrm{NiP}$ foams using tomographic images and stereoscopic micrographs. International journal of heat and mass transfer, 55(5), 1606-1619.

[26] Coquard, R., Rochais, D., \& Baillis, D. (2011). Modeling of the Coupled Conductive and Radiative Heat Transfer in Nicral from Photothermal Measurements and X-Ray Tomography. Special Topics \& Reviews in Porous Media, 2(4), 249-265.

[27] Coquard, R., Baillis, D., \& Maire, E. (2010). Numerical investigation of the radiative properties of polymeric foams from tomographic images. Journal of Thermophysics and Heat Transfer, 24(3), 647-658.

[28] Cunsolo, S., Coquard, R., Baillis, D., \& Bianco, N. (2016). Radiative properties modeling of open cell solid foam: Review and new analytical law. International Journal of Thermal Sciences, 104, 122-134.

[29] Akolkar, A., \& Petrasch, J. (2011). Tomography based pore-level optimization of radiative transfer in porous media. International Journal of Heat and Mass Transfer, 54(23), 4775-4783.

[30] Suter, S., Steinfeld, A., \& Haussener, S. (2014). Pore-level engineering of macroporous media for increased performance of solar-driven thermochemical fuel processing. International Journal of Heat and Mass Transfer, 78, 688-698. 
[31] Dyck, N. J., \& Straatman, A. G. (2015). A new approach to digital generation of spherical void phase porous media microstructures. International Journal of Heat and Mass Transfer, 81, 470477.

[32] Cunsolo, S., Oliviero, M., Harris, W. M., Andreozzi, A., Bianco, N., Chiu, W. K., \& Naso, V. (2015). Monte Carlo determination of radiative properties of metal foams: Comparison between idealized and real cell structures. International Journal of Thermal Sciences, 87, 94-102.

[33] Kumar, P., Topin, F., \& Vicente, J. (2014). Determination of effective thermal conductivity from geometrical properties: Application to open cell foams. International Journal of Thermal Sciences, 81, 13-28.

[34] Coquard, R., Randrianalisoa, J. H., \& Baillis, D. (2013). Computational prediction of radiative properties of polymer closed-cell foams with random structure. Journal of Porous Media, 16(2).

[35] Rousseau, B., Guevelou, S., Domingues, G., Vicente, J., Caliot, C., \& Flamant, G. (2013). Prediction of the radiative properties of reconstructed alpha-SiC foams used for concentrated solar applications. MRS Online Proceedings Library, 1545, mrss13-1545.

[36] Randrianalisoa, J., Coquard, R., \& Baillis, D. (2013). Microscale direct calculation of solid phase conductivity of voronoi s foams. Journal of Porous Media, 16, 411-426.

[37] Brakke, K. A. (1992). The surface evolver. Experimental mathematics, 1(2), 141-165.

[38] Kobbelt, L. P., Vorsatz, J., \& Labsik, U. (1999). A shrink wrapping approach to remeshing polygonal surfaces. In Computer Graphics Forum (Vol. 18, No. 3, pp. 119-130).

[39] Kraynik, A. M., Reinelt, D. A., \& van Swol, F. (2003). Structure of random monodisperse foam. Physical Review E, 67(3), 031403-1/11.

[40] Brun, E., Vicente, J., Topin, F. and Occelli, R., 2008. IMorph: A 3D morphological tool to fully analyse all kind of cellular materials. Cellular Metals for Structural and Functional Applications.

[41] Loretz, M., Maire, E., \& Baillis, D. (2008). Analytical Modelling of the Radiative Properties of Metallic Foams: Contribution of X-Ray Tomography. Advanced Engineering Materials, 10(4), 352-360.

[42] Ranut, P., Nobile, E., \& Mancini, L. (2014). High resolution microtomography-based CFD simulation of flow and heat transfer in aluminum metal foams. Applied Thermal Engineering, 69(1), 230-240.

[43] Glicksman, L., Schuetz, M., \& Sinofsky, M. (1987). Radiation heat transfer in foam insulation. International Journal of Heat and Mass Transfer, 30(1), 187-197.

[44] Russ, J. C., \& Dehoff, R. T. (2012). Practical stereology. Springer Science \& Business Media.

[45] Glicksman, Leon R., and M. R. Torpey. A study of radiative heat transfer through foam insulation. No. ORNL/Sub-86-09099/3. Oak Ridge National Lab., TN (USA); Massachusetts Inst. of Tech., Cambridge (USA), 1988.

[46] Kuhn, J., Ebert, H. P., Arduini-Schuster, M. C., Büttner, D., \& Fricke, J. (1992). Thermal transport in polystyrene and polyurethane foam insulations. International Journal of Heat and Mass Transfer, 35(7), 1795-1801. 